\title{
Vertex subsets with minimal width and dual width in $Q$-polynomial distance-regular graphs
}

\author{
Hajime Tanaka* \\ Department of Mathematics, University of Wisconsin \\ 480 Lincoln Drive, Madison, WI 53706, U.S.A. \\ htanaka@math.is.tohoku.ac.jp \\ Submitted: Nov 8, 2010; Accepted: Aug 4, 2011; Published: Aug 19, 2011 \\ Mathematics Subject Classifications: 05E30, 06A12
}

\begin{abstract}
We study $Q$-polynomial distance-regular graphs from the point of view of what we call descendents, that is to say, those vertex subsets with the property that the width $w$ and dual width $w^{*}$ satisfy $w+w^{*}=d$, where $d$ is the diameter of the graph. We show among other results that a nontrivial descendent with $w \geqslant 2$ is convex precisely when the graph has classical parameters. The classification of descendents has been done for the 5 classical families of graphs associated with short regular semilattices. We revisit and characterize these families in terms of posets consisting of descendents, and extend the classification to all of the 15 known infinite families with classical parameters and with unbounded diameter.
\end{abstract}

\section{Introduction}

Q-polynomial distance-regular graphs are thought of as finite/combinatorial analogues of compact symmetric spaces of rank one, and are receiving considerable attention; see e.g., $[2,3,15,25]$ and the references therein. In this paper, we study these graphs further from the point of view of what we shall call descendents, that is to say, those (vertex) subsets with the property that the width $w$ and dual width $w^{*}$ satisfy $w+w^{*}=d$, where $d$ is the diameter of the graph. See $\S 2$ for formal definitions. A typical example is a $w$-cube $H(w, 2)$ in the $d$-cube $H(d, 2)(w \leqslant d)$.

The width and dual width of subsets were introduced and discussed in detail by Brouwer, Godsil, Koolen and Martin [4], and descendents arise as a special, but very important, case of the theory $[4, \S 5]$. They showed among other results that every descendent

*Regular address: Graduate School of Information Sciences, Tohoku University, 6-3-09 Aramaki-AzaAoba, Aoba-ku, Sendai 980-8579, Japan 
is completely regular, and that the induced subgraph is a $Q$-polynomial distance-regular graph if it is connected [4, Theorems 1-3]. When the graph is defined on the top fiber of a short regular semilattice [13] (as is the case for the $d$-cube), each object of the semilattice naturally gives rise to a descendent [4, Theorem 5]. Hence we may also view descendents as reflecting intrinsic geometric structures of $Q$-polynomial distance-regular graphs. Incidentally, descendents have been applied to the Erdős-Ko-Rado theorem in extremal set theory [31, Theorem 3], and implicitly to the Assmus-Mattson theorem in coding theory [32, Examples 5.4, 5.5].

Associated with each $Q$-polynomial distance-regular graph $\Gamma$ is a Leonard system [38, $39,40]$, a linear algebraic framework for a famous theorem of Leonard [24], [2, §3.5] which characterizes the terminating branch of the Askey scheme [21] of (basic) hypergeometric orthogonal polynomials ${ }^{1}$ by the duality properties of $\Gamma$. The starting point of the research presented in this paper is a result of Hosoya and Suzuki [20, Proposition 1.3] which gives a system of linear equations satisfied by the eigenmatrix of the induced subgraph $\Gamma_{Y}$ of a descendent $Y$ of $\Gamma$ (when it is connected), and we reformulate this result as the existence of a balanced bilinear form between the underlying vector spaces of the Leonard systems associated with $\Gamma$ and $\Gamma_{Y}$; see $\S 4$. Balanced bilinear forms were independently studied in detail in an earlier paper [33], and we may derive all the parametric information on descendents from the results of [33].

The contents of the paper are as follows. Sections 2 and 3 review basic notation, terminology and facts concerning $Q$-polynomial distance-regular graphs and Leonard systems. The concept of a descendent is introduced in $\S 2$. In $\S 4$, we relate descendents and balanced bilinear forms. We give a necessary and sufficient condition on $\Gamma_{Y}$ to be $Q$ polynomial distance-regular (or equivalently, to be connected) in terms of the parameters of $\Gamma$ (Proposition 4.2). In passing, we also show that if $\Gamma_{Y}$ is connected then a nonempty subset of $Y$ is a descendent of $\Gamma_{Y}$ precisely when it is a descendent of $\Gamma$ (Proposition 4.4), so that we may define a poset structure on the set of isomorphism classes of $Q$-polynomial distance-regular graphs in terms of isometric embeddings as descendents. It should be remarked that the parameters of $\Gamma_{Y}$ in turn determine those of $\Gamma$, provided that the width of $Y$ is at least three; see Proposition 4.3.

In $\S 5$, we suppose $\Gamma$ is bipartite (with diameter $d$ ). The induced subgraph $\Gamma_{d}^{2}(x)$ of the distance-2 graph of $\Gamma$ on the set $\Gamma_{d}(x)$ of vertices at distance $d$ from a fixed vertex $x$ is known [8] to be distance-regular and $Q$-polynomial. We show that if $\Gamma_{d}^{2}(x)$ has diameter $\lfloor d / 2\rfloor$ then for every descendent $Y$ of a halved graph of $\Gamma, Y \cap \Gamma_{d}(x)$ is a descendent of $\Gamma_{d}^{2}(x)$ unless it is empty (Proposition 5.2). This result will be used in $\S 8$.

Section 6 establishes the main results of the present paper. Many classical examples of $Q$-polynomial distance-regular graphs have the property that their parameters are expressed in terms of the diameter $d$ and three other parameters $q, \alpha, \beta$ [3, p. 193]. Such graphs are said to have classical parameters $(d, q, \alpha, \beta)$. There are many results characterizing this property in terms of substructures of graphs; see e.g., [42, Theorem 7.2], [41]. We show that a nontrivial descendent $Y$ with width $w \geqslant 2$ is convex (i.e., geodetically closed) precisely when $\Gamma$ has classical parameters $(d, q, \alpha, \beta)$ (Theorem 6.3). Moreover, if

\footnotetext{
${ }^{1}$ We also allow the specialization $q \rightarrow-1$.
} 
this is the case then $\Gamma_{Y}$ has classical parameters $(w, q, \alpha, \beta)$ (Theorem 6.4).

In view of this connection with convexity, the remainder of the paper is concerned with graphs with classical parameters. Currently, there are 15 known infinite families of such graphs with unbounded diameter, and 5 of them are associated with short regular semilattices. The classification of descendents has been done for these 5 families; by Brouwer et al. [4, Theorem 8] for Johnson and Hamming graphs, and by the author [31, Theorem 1] for Grassmann, bilinear forms and dual polar graphs. It turned out that every descendent is isomorphic (under the full automorphism group of the graph) to one afforded by an object of the semilattice.

Section 7 is concerned with the 5 families of "semilattice-type" graphs. We show that if $d \geqslant 4$ then these graphs are characterized by the following properties: (1) $\Gamma$ has classical parameters; and there is a family $\mathscr{P}$ of descendents of $\Gamma$ such that (2) any two vertices, say, at distance $i$, are contained in a unique descendent in $\mathscr{P}$ with width $i$; and (3) the intersection of two descendents in $\mathscr{P}$ is either empty or a member of $\mathscr{P}$ (Theorem 7.19). We remark that if $\mathscr{P}$ is the set of descendents of $\Gamma$ then (1), (2) imply (3) (Proposition 7.20). We shall in fact show that $\mathscr{P}$, together with the partial order defined by reverse inclusion, forms a regular quantum matroid [37]. The semilattice structure of $\Gamma$ is then completely recovered from $\mathscr{P}$, and the characterization of $\Gamma$ follows from the classification of nontrivial regular quantum matroids with rank at least four [37, Theorem 39.6].

Section 8 extends the classification of descendents to all of the 15 families. We make heavy use of previous work on (noncomplete) convex subgraphs [23, 26] and maximal cliques $[16,17,5]$ in some of these families. We shall see a strong contrast between the distributions of descendents in the 5 families of "semilattice-type" and the other 10 families of "non-semilattice-type".

The paper ends with an appendix containing necessary data involving the parameter arrays (see $\S 3$ for the definition) of Leonard systems.

\section{$2 \quad Q$-polynomial distance-regular graphs}

Let $X$ be a finite set and $\mathbb{C}^{X \times X}$ the $\mathbb{C}$-algebra of complex matrices with rows and columns indexed by $X$. Let $\mathcal{R}=\left\{R_{0}, R_{1}, \ldots, R_{d}\right\}$ be a set of nonempty symmetric binary relations on $X$. For each $i$, let $A_{i} \in \mathbb{C}^{X \times X}$ be the adjacency matrix of the graph $\left(X, R_{i}\right)$. The pair $(X, \mathcal{R})$ is a (symmetric) association scheme with $d$ classes if

(AS1) $A_{0}=I$, the identity matrix;

(AS2) $\sum_{i=0}^{d} A_{i}=J$, the all ones matrix;

(AS3) $A_{i} A_{j} \in \boldsymbol{A}:=\left\langle A_{0}, A_{1}, \ldots, A_{d}\right\rangle$ for $0 \leqslant i, j \leqslant d$.

It follows from (AS1)-(AS3) that the $(d+1)$-dimensional vector space $\boldsymbol{A}$ is a commutative algebra, called the Bose-Mesner algebra of $(X, \mathcal{R})$. Since $\boldsymbol{A}$ is semisimple ${ }^{2}$ (as it is closed under conjugate-transposition), there is a basis $\left\{E_{i}\right\}_{i=0}^{d}$ consisting of the primitive

\footnotetext{
${ }^{2}$ We refer to $[11, \S 3]$ for the background material on semisimple algebras.
} 
idempotents of $\boldsymbol{A}$, i.e., $E_{i} E_{j}=\delta_{i j} E_{i}, \sum_{i=0}^{d} E_{i}=I$. We shall always set $E_{0}=|X|^{-1} J$. By (AS2), $\boldsymbol{A}$ is also closed under entrywise multiplication, denoted o. The $A_{i}$ are the primitive idempotents of $\boldsymbol{A}$ with respect to this multiplication, i.e., $A_{i} \circ A_{j}=\delta_{i j} A_{i}$, $\sum_{i=0}^{d} A_{i}=J$. For convenience, define $A_{i}=E_{i}=0$ if $i<0$ or $i>d$.

Let $\mathbb{C}^{X}$ be the Hermitean space of complex column vectors with coordinates indexed by $X$, so that $\mathbb{C}^{X \times X}$ acts on $\mathbb{C}^{X}$ from the left. For each $x \in X$ let $\hat{x}$ be the vector in $\mathbb{C}^{X}$ with a 1 in coordinate $x$ and 0 elsewhere. The $\hat{x}$ form an orthonormal basis for $\mathbb{C}^{X}$.

We say $(X, \mathcal{R})$ is $P$-polynomial with respect to the ordering $\left\{A_{i}\right\}_{i=0}^{d}$ if there are integers $a_{i}, b_{i}, c_{i}(0 \leqslant i \leqslant d)$ such that $b_{d}=c_{0}=0, b_{i-1} c_{i} \neq 0(1 \leqslant i \leqslant d)$ and

$$
A_{1} A_{i}=b_{i-1} A_{i-1}+a_{i} A_{i}+c_{i+1} A_{i+1} \quad(0 \leqslant i \leqslant d)
$$

where $b_{-1}=c_{d+1}=0$. Such an ordering is called a $P$-polynomial ordering. It follows that $\left(X, R_{1}\right)$ is regular of valency $k:=b_{0}, a_{i}+b_{i}+c_{i}=k(0 \leqslant i \leqslant d), a_{0}=0$ and $c_{1}=1$. Note that $A:=A_{1}$ generates $\boldsymbol{A}$ and hence has $d+1$ distinct eigenvalues $\theta_{0}:=k, \theta_{1}, \ldots, \theta_{d}$ so that $A=\sum_{i=0}^{d} \theta_{i} E_{i}$. Note also that $\left(X, R_{i}\right)$ is the distance- $i$ graph of $\left(X, R_{1}\right)$ for all $i$. Dually, we say $(X, \mathcal{R})$ is $Q$-polynomial with respect to the ordering $\left\{E_{i}\right\}_{i=0}^{d}$ if there are scalars $a_{i}^{*}, b_{i}^{*}, c_{i}^{*}(0 \leqslant i \leqslant d)$ such that $b_{d}^{*}=c_{0}^{*}=0, b_{i-1}^{*} c_{i}^{*} \neq 0(1 \leqslant i \leqslant d)$ and

$$
E_{1} \circ E_{i}=|X|^{-1}\left(b_{i-1}^{*} E_{i-1}+a_{i}^{*} E_{i}+c_{i+1}^{*} E_{i+1}\right) \quad(0 \leqslant i \leqslant d)
$$

where $b_{-1}^{*}=c_{d+1}^{*}=0$. Such an ordering is called a $Q$-polynomial ordering. It follows that $\operatorname{rank} E_{1}=m:=b_{0}^{*}, a_{i}^{*}+b_{i}^{*}+c_{i}^{*}=m(0 \leqslant i \leqslant d), a_{0}^{*}=0$ and $c_{1}^{*}=1$. Note that $|X| E_{1}$ generates $\boldsymbol{A}$ with respect to $\circ$ and hence has $d+1$ distinct entries $\theta_{0}^{*}:=m, \theta_{1}^{*}, \ldots, \theta_{d}^{*}$ so that $|X| E_{1}=\sum_{i=0}^{d} \theta_{i}^{*} A_{i}$. We may remark that

$$
\left\langle\left(E_{1} \mathbb{C}^{X}\right) \circ\left(E_{i} \mathbb{C}^{X}\right)\right\rangle \subseteq E_{i-1} \mathbb{C}^{X}+E_{i} \mathbb{C}^{X}+E_{i+1} \mathbb{C}^{X} \quad(0 \leqslant i \leqslant d) .
$$

See e.g., [2, p. 126, Proposition 8.3].

A connected simple graph $\Gamma$ with vertex set $V \Gamma=X$, diameter $d$ and path-length distance $\partial$ is called distance-regular if the distance- $i$ relations $(0 \leqslant i \leqslant d)$ together form an association scheme. Hence $P$-polynomial association schemes, with specified $P$ polynomial ordering, are in bijection with distance-regular graphs, and we shall say, e.g., that $\Gamma$ is $Q$-polynomial, and so on. The sequence

$$
\iota(\Gamma)=\left\{b_{0}, b_{1}, \ldots, b_{d-1} ; c_{1}, c_{2}, \ldots, c_{d}\right\}
$$

is called the intersection array of $\Gamma$. Given $x \in X$, we write $\Gamma_{i}(x)=\{y \in X: \partial(x, y)=i\}$, $k_{i}=\left|\Gamma_{i}(x)\right|(0 \leqslant i \leqslant d)$. We abbreviate $\Gamma(x)=\Gamma_{1}(x)$.

We say $\Gamma$ has classical parameters $(d, q, \alpha, \beta)[3$, p. 193] if

$$
b_{i}=\left(\left[\begin{array}{l}
d \\
1
\end{array}\right]_{q}-\left[\begin{array}{l}
i \\
1
\end{array}\right]_{q}\right)\left(\beta-\alpha\left[\begin{array}{l}
i \\
1
\end{array}\right]_{q}\right), \quad c_{i}=\left[\begin{array}{l}
i \\
1
\end{array}\right]_{q}\left(1+\alpha\left[\begin{array}{c}
i-1 \\
1
\end{array}\right]_{q}\right) \quad(0 \leqslant i \leqslant d)
$$

where $\left[\begin{array}{l}i \\ j\end{array}\right]_{q}$ is the $q$-binomial coefficient. By $[3$, Proposition 6.2.1], $q$ is an integer $\neq 0,-1$. In this case $\Gamma$ has a $Q$-polynomial ordering $\left\{E_{i}\right\}_{i=0}^{d}$ which we call standard, such that

$$
\theta_{i}^{*}=\xi^{*}\left[\begin{array}{c}
d-i \\
1
\end{array}\right]_{q}+\zeta^{*} \quad(0 \leqslant i \leqslant d)
$$


for some $\xi^{*}, \zeta^{*}$ with $\xi^{*} \neq 0[3$, Corollary 8.4.2].

For the rest of this section, suppose further that $\Gamma$ is $Q$-polynomial with respect to the ordering $\left\{E_{i}\right\}_{i=0}^{d}$. For the moment fix a "base vertex" $x \in X$, and let $E_{i}^{*}=E_{i}^{*}(x):=$ $\operatorname{diag}\left(A_{i} \hat{x}\right), A_{i}^{*}=A_{i}^{*}(x):=|X| \operatorname{diag}\left(E_{i} \hat{x}\right)(0 \leqslant i \leqslant d) .{ }^{3}$ We abbreviate $A^{*}=A_{1}^{*}$. Note that $E_{i}^{*} E_{j}^{*}=\delta_{i j} E_{i}^{*}, \sum_{i=0}^{d} E_{i}^{*}=I$. The $E_{i}^{*}$ and the $A_{i}^{*}$ form two bases for the dual Bose-Mesner algebra $\boldsymbol{A}^{*}=\boldsymbol{A}^{*}(x)$ with respect to $x$. Note also that $A^{*}$ generates $\boldsymbol{A}^{*}$ and $A^{*}=\sum_{i=0}^{d} \theta_{i}^{*} E_{i}^{*}$. The Terwilliger (or subconstituent) algebra $\boldsymbol{T}=\boldsymbol{T}(x)$ of $\Gamma$ with respect to $x$ is the subalgebra of $\mathbb{C}^{X \times X}$ generated by $\boldsymbol{A}, \boldsymbol{A}^{*}[34,35,36]$. Since $\boldsymbol{T}$ is closed under conjugate-transposition, it is semisimple and any two nonisomorphic irreducible $\boldsymbol{T}$-modules in $\mathbb{C}^{X}$ are orthogonal.

Let $Y$ be a nonempty subset of $X$ and $\hat{Y}=\sum_{x \in Y} \hat{x}$ its characteristic vector. We let $\Gamma_{Y}$ denote the subgraph of $\Gamma$ induced on $Y$. Set $Y_{i}=\{x \in X: \partial(x, Y)=i\}(0 \leqslant i \leqslant \rho)$, where $\rho=\max \{\partial(x, Y): x \in X\}$ is the covering radius of $Y$. Note that $\sum_{i=0}^{\rho} \hat{Y}_{i}=X$. We call $Y$ completely regular if $\left\langle\hat{Y}_{0}, \hat{Y}_{1}, \ldots, \hat{Y}_{\rho}\right\rangle$ is an $\boldsymbol{A}$-module. Brouwer et al. [4] defined the width $w$ and dual width $w^{*}$ of $Y$ as follows:

$$
w=\max \left\{i: \hat{Y}^{\top} A_{i} \hat{Y} \neq 0\right\}, \quad w^{*}=\max \left\{i: \hat{Y}^{\top} E_{i} \hat{Y} \neq 0\right\} .
$$

They showed (among other results) that

(2.8) Theorem $([4, \S 5])$. We have $w+w^{*} \geqslant d$. If equality holds then $Y$ is completely regular with covering radius $w^{*}$, and $\left(Y, \mathcal{R}^{Y}\right)$ forms a $Q$-polynomial association scheme with w-classes, where $\mathcal{R}^{Y}=\left\{R_{i} \cap(Y \times Y): 0 \leqslant i \leqslant w\right\}$.

We call $Y$ a descendent of $\Gamma$ if $w+w^{*}=d$. The descendents with $w=0$ are precisely the singletons, and $X$ is the unique descendent with $w=d$; we shall refer to these cases as trivial and say nontrivial otherwise. By (2.8) it follows that

(2.9) Theorem([4, Theorem 3]). Suppose $w+w^{*}=d$. If $\Gamma_{Y}$ is connected then it is a $Q$-polynomial distance-regular graph with diameter $w$.

We comment on the $Q$-polynomiality of $\left(Y, \mathcal{R}^{Y}\right)$ stated in (2.8). Suppose $w+w^{*}=d$ and let $\boldsymbol{A}^{\prime}$ be the Bose-Mesner algebra of $\left(Y, \mathcal{R}^{Y}\right)$. For every $B \in \boldsymbol{A}$, let $\breve{B}$ be the principal submatrix of $B$ corresponding to $Y$. Brouwer et al. [4, §4] observed

(2.10) $\breve{E}_{i} \breve{E}_{j}=0$ if $|i-j|>w^{*}$

and then showed that $\left\langle\breve{E}_{0}, \breve{E}_{1}, \ldots, \breve{E}_{i}\right\rangle$ is an ideal of $\boldsymbol{A}^{\prime}$ for all $i$. Hence we get a $Q$ polynomial ordering $\left\{E_{i}^{\prime}\right\}_{i=0}^{w}$ of the primitive idempotents of $\boldsymbol{A}^{\prime}$ such that

$$
\left\langle E_{0}^{\prime}, E_{1}^{\prime}, \ldots, E_{i}^{\prime}\right\rangle=\left\langle\breve{E}_{0}, \breve{E}_{1}, \ldots, \breve{E}_{i}\right\rangle \quad(0 \leqslant i \leqslant w) .
$$

Throughout we shall adopt the following convention and retain the notation of $\S 2$ :

(2.12) For the rest of this paper, we assume $\Gamma$ is distance-regular with diameter $d \geqslant 3$ and is $Q$-polynomial with respect to the ordering $\left\{E_{i}\right\}_{i=0}^{d}$. Unless otherwise stated, $Y$ will denote a nontrivial descendent of $\Gamma$ with width $w$ and dual width $w^{*}=d-w$.

\footnotetext{
${ }^{3}$ For a complex matrix $B$, it is customary that $B^{*}$ denotes the conjugate transpose of $B$. It should be stressed that we are not using this convention.
} 


\section{Leonard systems}

Let $d$ be a positive integer. Let $\mathfrak{A}$ be a $\mathbb{C}$-algebra isomorphic to the full matrix algebra $\mathbb{C}^{(d+1) \times(d+1)}$ and $W$ an irreducible left $\mathfrak{A}$-module. Note that $W$ is unique up to isomorphism and $\operatorname{dim} W=d+1$. An element $\mathfrak{a}$ of $\mathfrak{A}$ is called multiplicity-free if it has $d+1$ mutually distinct eigenvalues. Suppose $\mathfrak{a}$ is multiplicity-free and let $\left\{\theta_{i}\right\}_{i=0}^{d}$ be an ordering of the eigenvalues of $\mathfrak{a}$. Then there is a sequence of elements $\left\{\mathfrak{e}_{i}\right\}_{i=0}^{d}$ in $\mathfrak{A}$ such that (i) $\mathfrak{a} \mathfrak{e}_{i}=\theta_{i} \mathfrak{e}_{i}$; (ii) $\mathfrak{e}_{i} \mathfrak{e}_{j}=\delta_{i j} \mathfrak{e}_{i}$; (iii) $\sum_{i=0}^{d} \mathfrak{e}_{i}=\mathbf{1}$ where $\mathbf{1}$ is the identity of $\mathfrak{A}$. We call $\mathfrak{e}_{i}$ the primitive idempotent of $\mathfrak{a}$ associated with $\theta_{i}$. Note that $\mathfrak{a}$ generates $\left\langle\mathfrak{e}_{0}, \mathfrak{e}_{1}, \ldots, \mathfrak{e}_{d}\right\rangle$.

A Leonard system in $\mathfrak{A}[38$, Definition 1.4] is a sequence

(3.1) $\Phi=\left(\mathfrak{a} ; \mathfrak{a}^{*} ;\left\{\mathfrak{e}_{i}\right\}_{i=0}^{d} ;\left\{\mathfrak{e}_{i}^{*}\right\}_{i=0}^{d}\right)$

satisfying the following axioms (LS1)-(LS5):

(LS1) Each of $\mathfrak{a}, \mathfrak{a}^{*}$ is a multiplicity-free element in $\mathfrak{A}$.

(LS2) $\left\{\mathfrak{e}_{i}\right\}_{i=0}^{d}$ is an ordering of the primitive idempotents of $\mathfrak{a}$.

(LS3) $\left\{\mathfrak{e}_{i}^{*}\right\}_{i=0}^{d}$ is an ordering of the primitive idempotents of $\mathfrak{a}^{*}$.

$(\mathrm{LS} 4) \mathfrak{e}_{i}^{*} \mathfrak{a} \mathfrak{e}_{j}^{*}=\left\{\begin{array}{ll}0 & \text { if }|i-j|>1 \\ \neq 0 & \text { if }|i-j|=1\end{array} \quad(0 \leqslant i, j \leqslant d)\right.$.

$(\mathrm{LS} 5) \mathfrak{e}_{i} \mathfrak{a}^{*} \mathfrak{e}_{j}=\left\{\begin{array}{ll}0 & \text { if }|i-j|>1 \\ \neq 0 & \text { if }|i-j|=1\end{array} \quad(0 \leqslant i, j \leqslant d)\right.$.

We call $d$ the diameter of $\Phi$. For convenience, define $\mathfrak{e}_{i}=\mathfrak{e}_{i}^{*}=0$ if $i<0$ or $i>d$. Observe

$$
\mathfrak{e}_{0}^{*} W+\mathfrak{e}_{1}^{*} W+\cdots+\mathfrak{e}_{i}^{*} W=\mathfrak{e}_{0}^{*} W+\mathfrak{a} \mathfrak{e}_{0}^{*} W+\cdots+\mathfrak{a}^{i} \mathfrak{e}_{0}^{*} W \quad(0 \leqslant i \leqslant d) .
$$

A Leonard system $\Psi$ in a $\mathbb{C}$-algebra $\mathfrak{B}$ is isomorphic to $\Phi$ if there is a $\mathbb{C}$-algebra isomorphism $\sigma: \mathfrak{A} \rightarrow \mathfrak{B}$ such that $\Psi=\Phi^{\sigma}:=\left(\mathfrak{a}^{\sigma} ; \mathfrak{a}^{* \sigma} ;\left\{\mathfrak{e}_{i}^{\sigma}\right\}_{i=0}^{d} ;\left\{\mathfrak{e}_{i}^{* \sigma}\right\}_{i=0}^{d}\right)$. Let $\xi, \xi^{*}, \zeta, \zeta^{*}$ be scalars with $\xi, \xi^{*} \neq 0$. Then

$$
\left(\xi \mathfrak{a}+\zeta \mathbf{1} ; \xi^{*} \mathfrak{a}^{*}+\zeta^{*} \mathbf{1} ;\left\{\mathfrak{e}_{i}\right\}_{i=0}^{d} ;\left\{\mathfrak{e}_{i}^{*}\right\}_{i=0}^{d}\right)
$$

is a Leonard system in $\mathfrak{A}$, called an affine transformation of $\Phi$. We say $\Phi, \Psi$ are affineisomorphic if $\Psi$ is isomorphic to an affine transformation of $\Phi$. The dual of $\Phi$ is

$$
\Phi^{*}=\left(\mathfrak{a}^{*} ; \mathfrak{a} ;\left\{\mathfrak{e}_{i}^{*}\right\}_{i=0}^{d} ;\left\{\mathfrak{e}_{i}\right\}_{i=0}^{d}\right) .
$$

For any object $f$ associated with $\Phi$, we shall occasionally denote by $f^{*}$ the corresponding object for $\Phi^{*}$; an example is $\mathfrak{e}_{i}^{*}(\Phi)=\mathfrak{e}_{i}\left(\Phi^{*}\right)$. Note that $\left(f^{*}\right)^{*}=f$.

(3.5) Example. With reference to (2.12), fix the base vertex $x \in X$ and let $W=\boldsymbol{A} \hat{x}=$ $\boldsymbol{A}^{*} \hat{X}$ be the primary $\boldsymbol{T}$-module [34, Lemma 3.6]. Set $\mathfrak{a}=\left.A\right|_{W}, \mathfrak{a}^{*}=\left.A^{*}\right|_{W}, \mathfrak{e}_{i}=\left.E_{i}\right|_{W}$, $\mathfrak{e}_{i}^{*}=\left.E_{i}^{*}\right|_{W}(0 \leqslant i \leqslant d)$. Then $\Phi=\Phi(\Gamma ; x):=\left(\mathfrak{a} ; \mathfrak{a}^{*} ;\left\{\mathfrak{e}_{i}\right\}_{i=0}^{d} ;\left\{\mathfrak{e}_{i}^{*}\right\}_{i=0}^{d}\right)$ is a Leonard system. See [35, Theorem 4.1], [10]. We remark that $\Phi(\Gamma ; x)$ does not depend on $x$ up to isomorphism, so that we shall write $\Phi(\Gamma)=\Phi(\Gamma ; x)$ where the context allows. 
(3.6) Example. More generally, let $W$ be any irreducible $\boldsymbol{T}$-module. We say $W$ is thin if $\operatorname{dim} E_{i}^{*} W \leqslant 1$ for all $i$. Suppose $W$ is thin. Then $W$ is dual thin, i.e., $\operatorname{dim} E_{i} W \leqslant 1$ for all $i$, and there are integers $\epsilon$ (endpoint), $\epsilon^{*}$ (dual endpoint), $\delta$ (diameter) such that $\left\{i: E_{i}^{*} W \neq 0\right\}=\{\epsilon, \epsilon+1, \ldots, \epsilon+\delta\},\left\{i: E_{i} W \neq 0\right\}=\left\{\epsilon^{*}, \epsilon^{*}+1, \ldots, \epsilon^{*}+\delta\right\}[34$, Lemmas $3.9,3.12] .{ }^{4} \quad$ Set $\mathfrak{a}=\left.A\right|_{W}, \mathfrak{a}^{*}=\left.A^{*}\right|_{W}, \mathfrak{e}_{i}=\left.E_{\epsilon^{*}+i}\right|_{W}, \mathfrak{e}_{i}^{*}=\left.E_{\epsilon+i}^{*}\right|_{W}(0 \leqslant i \leqslant \delta)$. Then $\Phi=\Phi(W):=\left(\mathfrak{a} ; \mathfrak{a}^{*} ;\left\{\mathfrak{e}_{i}\right\}_{i=0}^{\delta} ;\left\{\mathfrak{e}_{i}^{*}\right\}_{i=0}^{\delta}\right)$ is a Leonard system. Note that $\Phi(\Gamma ; x)=\Phi(\boldsymbol{A} \hat{x})$.

For $0 \leqslant i \leqslant d$ let $\theta_{i}\left(\right.$ resp. $\left.\theta_{i}^{*}\right)$ be the eigenvalue of $\mathfrak{a}\left(\right.$ resp. $\left.\mathfrak{a}^{*}\right)$ associated with $\mathfrak{e}_{i}$ (resp. $\left.\mathfrak{e}_{i}^{*}\right)$. By [38, Theorem 3.2] there are scalars $\varphi_{i}(1 \leqslant i \leqslant d)$ and a $\mathbb{C}$-algebra isomorphism $\natural: \mathfrak{A} \rightarrow \mathbb{C}^{(d+1) \times(d+1)}$ such that $\mathfrak{a}^{\natural}$ (resp. $\left.\mathfrak{a}^{* \natural}\right)$ is the lower (resp. upper) bidiagonal matrix with diagonal entries $\left(\mathfrak{a}^{\natural}\right)_{i i}=\theta_{i}$ (resp. $\left.\left(\mathfrak{a}^{* \natural}\right)_{i i}=\theta_{i}^{*}\right)(0 \leqslant i \leqslant d)$ and subdiagonal (resp. superdiagonal) entries $\left(\mathfrak{a}^{\natural}\right)_{i, i-1}=1$ (resp. $\left.\left(\mathfrak{a}^{* \natural}\right)_{i-1, i}=\varphi_{i}\right)(1 \leqslant i \leqslant d)$. We let $\phi_{i}=\varphi_{i}\left(\Phi^{\Downarrow}\right)$ $(1 \leqslant i \leqslant d)$, where $\Phi^{\Downarrow}=\left(\mathfrak{a} ; \mathfrak{a}^{*} ;\left\{\mathfrak{e}_{d-i}\right\}_{i=0}^{d} ;\left\{\mathfrak{e}_{i}^{*}\right\}_{i=0}^{d}\right) .{ }^{5}$ The parameter array of $\Phi$ is

$$
p(\Phi)=\left(\left\{\theta_{i}\right\}_{i=0}^{d} ;\left\{\theta_{i}^{*}\right\}_{i=0}^{d} ;\left\{\varphi_{i}\right\}_{i=1}^{d} ;\left\{\phi_{i}\right\}_{i=1}^{d}\right) \text {. }
$$

By [38, Theorem 1.9], the isomorphism class of $\Phi$ is determined by $p(\Phi)$. In $[40], p(\Phi)$ is given in closed form; see also (A.1). Note that the parameter array of (3.3) is given by

$$
\left(\left\{\xi \theta_{i}+\zeta\right\}_{i=0}^{d} ;\left\{\xi^{*} \theta_{i}^{*}+\zeta^{*}\right\}_{i=0}^{d} ;\left\{\xi \xi^{*} \varphi_{i}\right\}_{i=1}^{d} ;\left\{\xi \xi^{*} \phi_{i}\right\}_{i=1}^{d}\right)
$$

Let $u$ be a nonzero vector in $\mathfrak{e}_{0} W$. Then $\left\{\mathfrak{e}_{i}^{*} u\right\}_{i=0}^{d}$ is a basis for $W$ [39, Lemma 10.2]. Define the scalars $a_{i}, b_{i}, c_{i}(0 \leqslant i \leqslant d)$ by $b_{d}=c_{0}=0$ and

$$
\mathfrak{a} \mathfrak{e}_{i}^{*} u=b_{i-1} \mathfrak{e}_{i-1}^{*} u+a_{i} \mathfrak{e}_{i}^{*} u+c_{i+1} \mathfrak{e}_{i+1}^{*} u \quad(0 \leqslant i \leqslant d)
$$

where $b_{-1}=c_{d+1}=0$. By $[39$, Theorem 17.7] it follows that

$$
b_{i}=\varphi_{i+1} \frac{\tau_{i}^{*}\left(\theta_{i}^{*}\right)}{\tau_{i+1}^{*}\left(\theta_{i+1}^{*}\right)}, \quad c_{i}=\phi_{i} \frac{\eta_{d-i}^{*}\left(\theta_{i}^{*}\right)}{\eta_{d-i+1}^{*}\left(\theta_{i-1}^{*}\right)} \quad(0 \leqslant i \leqslant d)
$$

where $\theta_{-1}^{*}, \theta_{d+1}^{*}$ are indeterminates, $\varphi_{d+1}=\phi_{0}=0$ and

$$
\tau_{i}(\lambda)=\prod_{l=0}^{i-1}\left(\lambda-\theta_{l}\right), \quad \eta_{i}(\lambda)=\prod_{l=0}^{i-1}\left(\lambda-\theta_{d-l}\right) \quad(0 \leqslant i \leqslant d)
$$

(3.12) Example. Let $\Phi=\Phi(\Gamma)$ be as in (3.5). Then $b_{i}(\Gamma)=b_{i}(\Phi), b_{i}^{*}(\Gamma)=b_{i}^{*}(\Phi)$, $c_{i}(\Gamma)=c_{i}(\Phi), c_{i}^{*}(\Gamma)=c_{i}^{*}(\Phi)(0 \leqslant i \leqslant d)$. See [35, Theorem 4.1].

Let $\Phi^{\prime}=\left(\mathfrak{a}^{\prime} ; \mathfrak{a}^{* \prime} ;\left\{\mathfrak{e}_{i}^{\prime}\right\}_{i=0}^{d^{\prime}} ;\left\{\mathfrak{e}_{i}^{* \prime}\right\}_{i=0}^{d^{\prime}}\right)$ be another Leonard system with diameter $d^{\prime} \leqslant d$ and $W^{\prime}=W\left(\Phi^{\prime}\right)$ the vector space underlying $\Phi^{\prime}$. Given an integer $\rho\left(0 \leqslant \rho \leqslant d-d^{\prime}\right)$, a nonzero bilinear form $(\cdot \mid \cdot): W \times W^{\prime} \rightarrow \mathbb{C}$ is called $\rho$-balanced with respect to $\Phi, \Phi^{\prime}$ if

\footnotetext{
${ }^{4}$ In $[34,35,36], \epsilon$ and $\epsilon^{*}$ are called the dual endpoint and endpoint of $W$, respectively.

${ }^{5}$ Viewed as permutations on all Leonard systems, $*$ and $\Downarrow$ generate a dihedral group with 8 elements which plays a fundamental role in the theory of Leonard systems.
} 
(B1) $\left(\mathfrak{e}_{i}^{*} W \mid \mathfrak{e}_{j}^{* \prime} W^{\prime}\right)=0$ if $i-\rho \neq j \quad\left(0 \leqslant i \leqslant d, 0 \leqslant j \leqslant d^{\prime}\right)$;

(B2) $\left(\mathfrak{e}_{i} W \mid \mathfrak{e}_{j}^{\prime} W^{\prime}\right)=0$ if $i<j$ or $i>j+d-d^{\prime} \quad\left(0 \leqslant i \leqslant d, 0 \leqslant j \leqslant d^{\prime}\right)$.

We call $\Phi^{\prime}$ a $\rho$-descendent of $\Phi$ whenever such a form exists. The $\rho$-descendents of $\Phi$ are completely classified; see (A.3). In particular, by (A.2), (A.3), (3.8) it follows that

(3.13) Proposition. Let $d, d^{\prime}, \rho$ be integers such that $1 \leqslant d^{\prime} \leqslant d, 0 \leqslant \rho \leqslant d-d^{\prime}$. Then a Leonard system with diameter $d$ has at most one $\rho$-descendent with diameter $d^{\prime}$ up to affine isomorphism. Conversely, if $d^{\prime} \geqslant 3$ then a Leonard system with diameter $d^{\prime}$ is a $\rho$-descendent of at most one Leonard system with diameter d up to affine isomorphism.

\section{Basic results concerning descendents}

With reference to (2.12), we begin with the following observation (cf. [20, p. 73]):

(4.1) With the notation of $\S 2$, for any $i, j(0 \leqslant i \leqslant d, 0 \leqslant j \leqslant w)$ we have

$$
\breve{E}_{i} E_{j}^{\prime}= \begin{cases}0 & \text { if } i<j \text { or } i>j+w^{*} \\ \neq 0 & \text { if } i=j \text { or } i=j+w^{*} .\end{cases}
$$

Proof. By (2.10), (2.11) it follows that $\breve{E}_{i} \in\left\langle E_{i-w^{*}}^{\prime}, \ldots, E_{i}^{\prime}\right\rangle$, so that $\breve{E}_{i} E_{j}^{\prime}=0$ if $i<j$ or $i>j+w^{*}$. By (2.11) we also find $\breve{E}_{j} E_{j}^{\prime} \neq 0$. Note that $E_{j+w^{*} \circ} E_{j} \in\left\langle E_{w^{*}}, \ldots, E_{d}\right\rangle$ and the coefficient of $E_{w^{*}}$ in $E_{j+w^{*}} \circ E_{j}$ is nonzero. Hence trace $\left(\breve{E}_{j+w^{*}} \breve{E}_{j}\right)=\hat{Y}^{\top}\left(E_{j+w^{*}} \circ E_{j}\right) \hat{Y} \neq 0$. It follows that $\breve{E}_{j+w^{*}} \breve{E}_{j} \neq 0$ and therefore $\breve{E}_{j+w^{*}} E_{j}^{\prime} \neq 0$ by (2.10), (2.11).

As mentioned in the introduction, Hosoya and Suzuki translated (4.1) into a system of linear equations satisfied by the eigenmatrix of $\left(Y, \mathcal{R}^{Y}\right)$; see [20, Proposition 1.3]. We now show how descendents are related to balanced bilinear forms:

(4.2) Proposition. Pick any $x \in Y$, and let the parameter array of $\Phi=\Phi(\Gamma ; x)$ be given as in (A.1). Suppose $w>1$. Then $\Gamma_{Y}$ is a $Q$-polynomial distance-regular graph precisely for Cases I, IA, II, IIA, IIB, IIC; or Case III with $w^{*}$ even. If this is the case then the bilinear form $(\cdot \mid \cdot): \boldsymbol{A} \hat{x} \times \boldsymbol{A}^{\prime} \hat{x} \rightarrow \mathbb{C}$ defined by $\left(u \mid u^{\prime}\right)=u^{\top} u^{\prime}$ is 0-balanced with respect to $\Phi, \Phi\left(\Gamma_{Y} ; x\right)$.

Proof. Write $W=\boldsymbol{A} \hat{x}, W^{\prime}=\boldsymbol{A}^{\prime} \hat{x}$. Note that $\left(E_{i} W \mid E_{j}^{\prime} W^{\prime}\right)=0$ whenever $\breve{E}_{i} E_{j}^{\prime}=0$. Hence it follows from (4.1) that $\left(E_{i} W \mid E_{j}^{\prime} W^{\prime}\right)=0$ if $i<j$ or $i>j+w^{*}$. Suppose $\Gamma_{Y}$ is distance-regular. Then by these comments we find that $(\cdot \mid \cdot)$ is 0 -balanced with respect to $\Phi, \Phi\left(\Gamma_{Y} ; x\right)$. By virtue of (A.3), $w^{*}$ must be even if $p(\Phi)$ is of Case III. Conversely, suppose $p(\Phi)$ (and $w^{*}$ ) satisfies one of the cases mentioned in (4.2). Then by [33, Theorem 7.3] there is a Leonard system $\Phi^{\prime}=\left(\mathfrak{a}^{\prime} ; \mathfrak{a}^{* \prime} ;\left\{\mathfrak{e}_{i}^{\prime}\right\}_{i=0}^{w} ;\left\{\mathfrak{e}_{i}^{* \prime}\right\}_{i=0}^{w}\right)$ with $W\left(\Phi^{\prime}\right)=W^{\prime}$ such that $\mathfrak{e}_{i}^{\prime}=$ $\left.E_{i}^{\prime}\right|_{W^{\prime}}, \mathfrak{e}_{i}^{* \prime}=\left.E_{i}^{* \prime}\right|_{W^{\prime}}$ where $E_{i}^{* \prime}=\operatorname{diag}\left(\breve{A}_{i} \hat{x}\right)(0 \leqslant i \leqslant w)$. Note that $\left.\breve{A}\right|_{W^{\prime}} \in\left\langle\mathfrak{e}_{0}^{\prime}, \mathfrak{e}_{1}^{\prime}, \ldots, \mathfrak{e}_{w}^{\prime}\right\rangle$, so that $\left.\breve{A}\right|_{W^{\prime}}$ is a polynomial in $\mathfrak{a}^{\prime}$. Since $\breve{A} \mathfrak{e}_{0}^{* \prime} W^{\prime}=\langle\breve{A} \hat{x}\rangle=\mathfrak{e}_{1}^{* \prime} W^{\prime}$, it follows from (3.2) that $\left.\breve{A}\right|_{W^{\prime}}=\xi \mathfrak{a}^{\prime}+\zeta \mathbf{s}^{\prime}$ for some $\xi, \zeta \in \mathbb{C}$ with $\xi \neq 0$, where $\mathbf{1}^{\prime}$ is the identity operator on $W^{\prime}$. Hence $\left\langle E_{i}^{* \prime} \breve{A}^{i} \hat{x}\right\rangle=\mathfrak{e}_{i}^{* \prime}\left(\mathfrak{a}^{\prime}\right)^{i} \mathfrak{e}_{0}^{* \prime} W^{\prime}=\mathfrak{e}_{i}^{* \prime} W^{\prime}=\left\langle\breve{A}_{i} \hat{x}\right\rangle(\neq 0)$ for $0 \leqslant i \leqslant w$. In particular, $\Gamma_{Y}$ is connected and thus distance-regular by $(2.9)$. 
By (4.2), connectivity and therefore distance-regularity of $\Gamma_{Y}$ can be read off the parameters of $\Gamma$.

(4.3) Proposition. Suppose $\Gamma_{Y}$ is distance-regular. Then $\Phi\left(\Gamma_{Y}\right)$ is uniquely determined by $\Phi(\Gamma)$ up to isomorphism. Conversely, if $w \geqslant 3$ then $\Phi\left(\Gamma_{Y}\right)$ uniquely determines $\Phi(\Gamma)$ up to isomorphism.

Proof. By (4.2), $\Phi\left(\Gamma_{Y}\right)$ is a 0-descendent of $\Phi(\Gamma)$. Hence the result follows from (3.13) together with the additional normalizations $b_{0}(\Psi)=\theta_{0}(\Psi), b_{0}^{*}(\Psi)=\theta_{0}^{*}(\Psi), c_{1}(\Psi)=$ $c_{1}^{*}(\Psi)=1$ for each $\Psi \in\left\{\Phi(\Gamma), \Phi\left(\Gamma_{Y}\right)\right\}$.

The following is another consequence of (4.1):

(4.4) Proposition. Suppose $\Gamma_{Y}$ is distance-regular. Then a nonempty subset of $Y$ is a descendent of $\Gamma_{Y}$ if and only if it is a descendent of $\Gamma$.

Proof. Let $Z \subseteq Y$ have dual width $w^{* \prime}$ in $\Gamma_{Y}$. For $0 \leqslant i \leqslant w$, by (4.1) we find $\breve{E}_{i+w^{*}} \in$ $\left\langle E_{i}^{\prime}, \ldots, E_{w}^{\prime}\right\rangle$ and the coefficient of $E_{i}^{\prime}$ in $\breve{E}_{i+w^{*}}$ is nonzero. Since $\hat{Z}^{\top} E_{i} \hat{Z}=\hat{Z}^{\top} \breve{E}_{i} \hat{Z}$, it follows that $Z$ has dual width $w^{* \prime}+w^{*}$ in $\Gamma$.

(4.5) Remark. Let $\mathscr{L}$ be the set of isomorphism classes of $Q$-polynomial distance-regular graphs with diameter at least three. For two isomorphism classes $[\Gamma],[\Delta] \in \mathscr{L}$, write $[\Delta] \preccurlyeq[\Gamma]$ if $[\Delta]=\left[\Gamma_{Y}\right]$ for some descendent $Y$ of $\Gamma$. Then by (4.4) it follows that $\preccurlyeq$ is a partial order on $\mathscr{L}$. Determining all descendents of $\Gamma$ amounts to describing the order ideal generated by $[\Gamma]$. Conversely, given $[\Gamma] \in \mathscr{L}$, it is a problem of some significance to determine the filter generated by $[\Gamma]$, i.e., $V_{[\Gamma]}=\{[\Delta] \in \mathscr{L}:[\Gamma] \preccurlyeq[\Delta]\}$.

Let $A^{*}(Y)=|X||Y|^{-1} \operatorname{diag}\left(E_{1} \hat{Y}\right), E_{i}^{*}(Y)=\operatorname{diag}\left(\hat{Y}_{i}\right)\left(0 \leqslant i \leqslant w^{*}\right)$ where $Y_{i}=\{x \in X$ : $\partial(x, Y)=i\}$. Let $\tilde{W}=\boldsymbol{A} \hat{Y}=\left\langle\hat{Y}_{0}, \hat{Y}_{1}, \ldots, \hat{Y}_{w^{*}}\right\rangle$. Note that $A^{*}(Y) \tilde{W} \subseteq \tilde{W}$. Following [22, Definition 3.7], we call $Y$ Leonard (with respect to $\theta_{1}$ ) if the matrix representing $\left.A^{*}(Y)\right|_{\tilde{W}}$ with respect to the basis $\left\{E_{i} \hat{Y}\right\}_{i=0}^{w^{*}}$ for $\tilde{W}$ is irreducible ${ }^{6}$ tridiagonal. Set $\mathfrak{b}=\left.A\right|_{\tilde{W}}$, $\mathfrak{b}^{*}=\left.A^{*}(Y)\right|_{\tilde{W}}, \mathfrak{f}_{i}=\left.E_{i}\right|_{\tilde{W}}, \mathfrak{f}_{i}^{*}=\left.E_{i}^{*}(Y)\right|_{\tilde{W}}\left(0 \leqslant i \leqslant w^{*}\right)$. Then $Y$ is Leonard if and only if $\Phi(\Gamma ; Y):=\left(\mathfrak{b} ; \mathfrak{b}^{*} ;\left\{\mathfrak{f}_{i}\right\}_{i=0}^{w^{*}} ;\left\{\mathfrak{f}_{i}^{*}\right\}_{i=0}^{w^{*}}\right)$ is a Leonard system. The following is dual to (4.2):

(4.6) Proposition. Pick any $x \in Y$, and let the parameter array of $\Phi=\Phi(\Gamma ; x)$ be given as in (A.1). Suppose $w^{*}>1$. Then $Y$ is Leonard (with respect to $\theta_{1}$ ) precisely for Cases I, IA, II, IIA, IIB, IIC; or Case III with $w$ even. If this is the case then the bilinear form $(\cdot \mid \cdot): \boldsymbol{A} \hat{x} \times \boldsymbol{A} \hat{Y} \rightarrow \mathbb{C}$ defined by $\left(u \mid u^{\prime}\right)=u^{\top} u^{\prime}$ is 0 -balanced with respect to $\Phi^{*}, \Phi(\Gamma ; Y)^{*}$.

Proof. Note that $E_{i}^{*}(x) E_{j}^{*}(Y)=0$ whenever $i<j$ or $i>j+w$ (cf. (4.1)). Hence if $Y$ is Leonard then $(\cdot \mid \cdot)$ is 0 -balanced with respect to $\Phi^{*}, \Phi(\Gamma ; Y)^{*}$, so that by (A.3) it follows that $w$ must be even if $p(\Phi)$ is of Case III. ${ }^{7}$ Conversely, suppose $p(\Phi)$ (and $w$ ) satisfies one of the cases mentioned in (4.6). Then by [33, Theorem 7.3] there are operators $\mathfrak{c}, \mathfrak{c}^{*}$ on

\footnotetext{
${ }^{6} \mathrm{~A}$ tridiagonal matrix is irreducible [38] if all the superdiagonal and subdiagonal entries are nonzero.

${ }^{7}$ The permutation $*$ (see footnote 5 ) leaves each of Cases I, IA, II, IIC, III invariant and swaps Cases IIA and IIB.
} 
$\tilde{W}$ such that $\left(\mathfrak{c} ; \mathfrak{c}^{*} ;\left\{\mathfrak{f}_{i}\right\}_{i=0}^{w^{*}} ;\left\{\mathfrak{f}_{i}^{*}\right\}_{i=0}^{w^{*}}\right)$ is a Leonard system. Note that $\mathfrak{b}^{*} \in\left\langle\mathfrak{f}_{0}^{*}, \mathfrak{f}_{1}^{*}, \ldots, \mathfrak{f}_{w^{*}}^{*}\right\rangle$, so that $\mathfrak{b}^{*}$ is a polynomial in $\mathfrak{c}^{*}$. Since $\mathfrak{b}^{*} \mathfrak{f}_{0} \tilde{W}=\left\langle E_{1} \hat{Y}\right\rangle=\mathfrak{f}_{1} \tilde{W}$, it follows from (3.2) that $\mathfrak{b}^{*}=\xi^{*} \mathfrak{c}^{*}+\zeta^{*} \tilde{\mathbf{I}}$ for some $\xi^{*}, \zeta^{*} \in \mathbb{C}$ with $\xi^{*} \neq 0$, where $\tilde{\mathbf{I}}$ is the identity operator on $\tilde{W}$. Hence the matrix representing $\mathfrak{b}^{*}$ with respect to $\left\{E_{i} \hat{Y}\right\}_{i=0}^{w^{*}}$ is irreducible tridiagonal. In other words, $Y$ is Leonard, as desired.

(4.7) Remark. Suppose $\Gamma$ is a translation distance-regular graph [3, §11.1C] and $Y$ is also a subgroup of the abelian group $X$. Then by [22, Proposition 3.3, Theorem 3.10], $Y$ is Leonard if and only if the coset graph $\Gamma / Y$ is $Q$-polynomial. Hence (4.6) strengthens [4, Theorem 4], which states that $\Gamma / Y$ is $Q$-polynomial if it is primitive. Note that if $Y$ is Leonard then $\Phi(\Gamma / Y ; Y)$ (where $Y$ is a vertex of $\Gamma / Y)$ is affine isomorphic to $\Phi(\Gamma ; Y)$.

It seems that (4.6) also motivates further analysis of the Terwilliger algebra with respect to $Y$ in the sense of Suzuki [30]; this will be discussed elsewhere.

\section{The bipartite case}

(5.1) With reference to (2.12), in this section we further assume that $\Gamma$ is bipartite and $d \geqslant 6$ (so that the halved graphs have diameter at least three).

With reference to (5.1), fix $x \in X$ and let $\Gamma_{d}^{2}=\Gamma_{d}^{2}(x)$ be the graph with vertex set $\Gamma_{d}=\Gamma_{d}(x)$ and edge set $\left\{(y, z) \in \Gamma_{d} \times \Gamma_{d}: \partial(y, z)=2\right\}$. Caughman [8, Theorems 9.2, 9.6, Corollary 4.4] showed that $\Gamma_{d}^{2}$ is distance-regular and $Q$-polynomial with diameter $\mathfrak{d}$, where $\mathfrak{d}$ equals half the width of $\Gamma_{d}$. In this section, we shall prove a result relating descendents of a halved graph of $\Gamma$ to those of $\Gamma_{d}^{2}$; see (5.2) below.

Write $E_{i}^{*}=E_{i}^{*}(x)(0 \leqslant i \leqslant d)$ and $\boldsymbol{T}=\boldsymbol{T}(x)$. Let $W$ be an irreducible $\boldsymbol{T}$-module with endpoint $\epsilon$, dual endpoint $\epsilon^{*}$ and diameter $\delta$ (see (3.6)). By [7, Lemma 9.2, Theorem 9.4], $W$ is thin, dual thin and $2 \epsilon^{*}+\delta=d$. In particular, $0 \leqslant \epsilon^{*} \leqslant\lfloor d / 2\rfloor$ and $\epsilon \leqslant 2 \epsilon^{*}$. Let $U_{i j}$ be the sum of the irreducible $\boldsymbol{T}$-modules $W$ in $\mathbb{C}^{X}$ with $\epsilon=i$ and $\epsilon^{*}=j$. By [7, Theorem 13.1], the (nonzero) $U_{i j}$ are the homogeneous components of $\mathbb{C}^{X}$. Note that $E_{d}^{*} U_{i j}=0$ unless $i=2 j$, so that $E_{d}^{*} \mathbb{C}^{X}=\sum_{j=0}^{\lfloor d / 2\rfloor} E_{d}^{*} U_{2 j, j}$ (orthogonal direct sum). By [8, Theorem 9.2] $E_{d}^{*} \boldsymbol{A} E_{d}^{*}$ gives the Bose-Mesner algebra of $\Gamma_{d}^{2}$, and each of $E_{d}^{*} U_{2 j, j}(0 \leqslant j \leqslant\lfloor d / 2\rfloor)$ is a (not necessarily maximal) eigenspace for $E_{d}^{*} \boldsymbol{A} E_{d}^{*}$.

We now compute the eigenvalue of the adjacency matrix $E_{d}^{*} A_{2} E_{d}^{*}$ of $\Gamma_{d}^{2}$ on $E_{d}^{*} U_{2 j, j}$. If $\Gamma$ is the $d$-cube $H(d, 2)$ then $\Gamma_{d}$ is a singleton and there is nothing to discuss. Suppose $\Gamma$ is the folded cube $\bar{H}(2 d, 2)$. Let $W \subseteq U_{2 j, j}$ and let $\Phi=\Phi(W)$ be as in (3.6). By [36, Example 6.1], $a_{i}(\Phi)=0(0 \leqslant i \leqslant \delta), b_{i}(\Phi)=2 \delta-i(0 \leqslant i \leqslant \delta-1), c_{i}(\Phi)=i$ $(1 \leqslant i \leqslant \delta-1)$ and $c_{\delta}(\Phi)=2 \delta$, where $\delta=d-2 j$. Using $A^{2}=c_{2} A_{2}+k I$ we find that $E_{d}^{*} A_{2} E_{d}^{*}$ has eigenvalue $(d-2 j)^{2}-2 j$ on $E_{d}^{*} W$ (and hence on $E_{d}^{*} U_{2 j, j}$ ). Next suppose $\Gamma \neq H(d, 2), \bar{H}(2 d, 2)$. Then by [7, pp. 89-91], $p(\Phi(\Gamma))$ satisfies Case I in (A.1) and in this case there are scalars $q, s^{*} \in \mathbb{C}$ (independent of $j$ ) such that $a_{i}(\Phi)=0(0 \leqslant i \leqslant \delta)$,

$$
b_{i}(\Phi)=\frac{h\left(q^{\delta}-q^{i}\right)\left(1-s^{*} q^{4 j+i+1}\right)}{q^{\delta+j}\left(1-s^{*} q^{4 j+2 i+1}\right)}, \quad c_{i}(\Phi)=\frac{h\left(q^{i}-1\right)\left(1-s^{*} q^{4 j+\delta+i+1}\right)}{q^{\delta+j}\left(1-s^{*} q^{4 j+2 i+1}\right)}
$$


for $1 \leqslant i \leqslant \delta-1$, and $b_{0}(\Phi)=c_{\delta}(\Phi)=h\left(q^{-j}-q^{j-d}\right)$, where

$$
h=\frac{q^{d}\left(1-s^{*} q^{3}\right)}{(q-1)\left(1-s^{*} q^{d+2}\right)} .
$$

Likewise we find that the eigenvalue of $E_{d}^{*} A_{2} E_{d}^{*}$ on $E_{d}^{*} U_{2 j, j}$ is given by

$$
\begin{aligned}
& \frac{1-s^{*} q^{5}}{\left(q^{2}-1\right)\left(1-s^{*} q^{d+2}\right)\left(1-s^{*} q^{d+3}\right)\left(1-s^{*} q^{2 d-1}\right) q} \\
& \times\left(\left(q^{d}-1\right)\left(q^{d}-q\right)\left(1-s^{* 2} q^{2 d+2}\right)+\frac{q^{2 d}\left(1-s^{*} q^{3}\right)\left(1-q^{2 j}\right)\left(1-s^{*} q^{2 j}\right)}{q^{2 j}}\right) .
\end{aligned}
$$

(5.2) Proposition. Referring to (5.1), suppose $\Gamma_{d}^{2}$ has diameter $\lfloor d / 2\rfloor$. If $Y$ is a descendent of a halved graph of $\Gamma$ with width $w$, then $Y \cap \Gamma_{d}$ is a descendent of $\Gamma_{d}^{2}$ with width $w$, provided that it is nonempty.

Proof. Note that $Y \cap \Gamma_{d}$ has width at most $w$ in $\Gamma_{d}^{2}$, and that the characteristic vector of $Y \cap \Gamma_{d}$ is $E_{d}^{*} \hat{Y}$. Let $w^{*}$ be the dual width of $Y$ in the halved graph. Then by [2, p. 328, Theorem 6.4] we find $\hat{Y} \in \sum_{j=0}^{w^{*}}\left(E_{j}+E_{d-j}\right) \mathbb{C}^{X}$, so that $E_{d}^{*} \hat{Y} \in \sum_{j=0}^{w^{*}} E_{d}^{*} U_{2 j, j}$. By assumption, $\Gamma \neq H(d, 2)$. Suppose $\Gamma=\bar{H}(2 d, 2)$. Then $\Gamma_{d}^{2}$ is the folded Johnson graph $\bar{J}(2 d, d)$. It follows (e.g., from [2, p. 301] or [36, Example 6.1]) that $(d-2 j)^{2}-2 j$ is the $j^{\text {th }}$ eigenvalue of $\bar{J}(2 d, d)$ in the $Q$-polynomial ordering $(0 \leqslant j \leqslant\lfloor d / 2\rfloor)$. Likewise, if $\Gamma \neq H(d, 2), \bar{H}(2 d, 2)$, then by the data in [8, p. 469], [2, pp. 264-265] we routinely find that the ordering $\left\{E_{d}^{*} U_{2 j, j}\right\}_{j=0}^{\lfloor d / 2\rfloor}$ of the eigenspaces of $\Gamma_{d}^{2}$ also agrees with the $Q$-polynomial ordering of $\Gamma_{d}^{2}$. Hence it follows that $Y \cap \Gamma_{d}$ has dual width at most $w^{*}$ in $\Gamma_{d}^{2}$. Since $\Gamma_{d}^{2}$ has diameter $\lfloor d / 2\rfloor=w+w^{*}$, we find that $Y \cap \Gamma_{d}$ is a descendent of $\Gamma_{d}^{2}$ and has width $w$, as desired.

\section{Convexity and graphs with classical parameters}

In this section, we shall prove our main results concerning convexity of descendents and classical parameters. Let $\Phi$ be the Leonard system from (3.1). By (3.10) we find

$$
\text { (6.1) } \frac{b_{i}(\Phi)}{c_{1}(\Phi)}=\frac{\varphi_{i+1} \eta_{d}^{*}\left(\theta_{0}^{*}\right) \tau_{i}^{*}\left(\theta_{i}^{*}\right)}{\phi_{1} \eta_{d-1}^{*}\left(\theta_{1}^{*}\right) \tau_{i+1}^{*}\left(\theta_{i+1}^{*}\right)}, \quad \frac{c_{i}(\Phi)}{c_{1}(\Phi)}=\frac{\phi_{i} \eta_{d}^{*}\left(\theta_{0}^{*}\right) \eta_{d-i}^{*}\left(\theta_{i}^{*}\right)}{\phi_{1} \eta_{d-1}^{*}\left(\theta_{1}^{*}\right) \eta_{d-i+1}^{*}\left(\theta_{i-1}^{*}\right)} \quad(0 \leqslant i \leqslant d) .
$$

Note that the values in (6.1) are invariant under affine transformation of $\Phi .{ }^{8}$ Moreover, if $\Phi=\Phi(\Gamma)$ then by (3.12) they coincide with $b_{i}(\Gamma)$ and $c_{i}(\Gamma)$, respectively, since $c_{1}(\Gamma)=1$. The following is a refinement of [3, Theorem 8.4.1], and is verified using (2.5), (2.6), (6.1):

(6.2) Proposition. With reference to (2.12), let the parameter array of $\Phi=\Phi(\Gamma)$ be given as in (A.1). Then $\Gamma$ has classical parameters if and only if $p(\Phi)$ satisfies either Case I with $s^{*}=0$; or Cases IA, IIA, IIC. If this is the case then $p(\Phi)$ and the classical parameters $(d, q, \alpha, \beta)$ are related as follows:

\footnotetext{
${ }^{8}$ Therefore, if $p(\Phi)$ satisfies, say, Case I in (A.1) then the resulting formulae involve $q, r_{1}, r_{2}, s, s^{*}$ only, and are independent of $h, h^{*}, \theta_{0}, \theta_{0}^{*}$.
} 


\begin{tabular}{cccc}
\hline Case & $q$ & $\alpha$ & $\beta$ \\
\hline I, $s^{*}=r_{1}=0$ & $q$ & $\frac{r_{2}(1-q)}{s q^{d}-r_{2}}$ & $\frac{r_{2} q-1}{q\left(s q^{d}-r_{2}\right)}$ \\
IA & $q$ & $\frac{r(1-q)}{s q^{d}-r}$ & $\frac{r}{s q^{d}-r}$ \\
IIA & 1 & $\frac{1}{r-s-d}$ & $\frac{-1-r}{r-s-d}$ \\
IIC & 1 & 0 & $\frac{-r}{r-s s^{*}}$ \\
\hline
\end{tabular}

Recall that $Y$ is called convex (or geodetically closed) if for any $x, y \in Y$ we have $\{z \in X: \partial(x, z)+\partial(z, y)=\partial(x, y)\} \subseteq Y$.

(6.3) Theorem. With reference to (2.12), suppose $1<w<d$. Then $Y$ is convex precisely when $\Gamma$ has classical parameters (with standard $Q$-polynomial ordering).

Proof. Let $\Phi=\Phi(\Gamma)$ and let $p(\Phi)$ be given as in (A.1). First, we may assume that $\Gamma_{Y}$ is distance-regular, or equivalently, by $(4.2)$, that $w^{*}$ is even if $p(\Phi)$ satisfies Case III. Indeed, if $Y$ is convex then $\Gamma_{Y}$ is connected and hence distance-regular by (2.9). On the other hand, if $\Gamma$ has classical parameters then by $(6.2), p(\Phi)$ does not satisfy Case III in the first place. Let $\Phi^{\prime}=\Phi\left(\Gamma_{Y}\right)$. Then, by (4.2), $p\left(\Phi^{\prime}\right)$ takes the form given in (A.3) with $\rho=0$. Note that $Y$ is convex if and only if $c_{i}(\Gamma)=c_{i}\left(\Gamma_{Y}\right)$ for all $1 \leqslant i \leqslant w$. Using (6.1) we find that $c_{i}(\Gamma) / c_{i}\left(\Gamma_{Y}\right)$ equals

$$
\begin{array}{ll}
\frac{\left(1-s^{*} q^{w+2}\right)\left(1-s^{*} q^{i+d+1}\right)}{\left(1-s^{*} q^{d+2}\right)\left(1-s^{*} q^{i+w+1}\right)} & \text { for Case I, } \\
\frac{\left(s^{*}+w+2\right)\left(s^{*}+i+d+1\right)}{\left(s^{*}+d+2\right)\left(s^{*}+i+w+1\right)} & \text { for Cases II, IIB, } \\
\frac{-s^{*}+w+2}{-s^{*}+d+2} & \text { for Case III, } d \text { even, } w \text { even, } i \text { even, } \\
\frac{\left(-s^{*}+w+2\right)\left(-s^{*}+i+d+1\right)}{\left(-s^{*}+d+2\right)\left(-s^{*}+i+w+1\right)} & \text { for Case III, } d \text { even, } w \text { even, } i \text { odd, } \\
\frac{-s^{*}+i+d+1}{-s^{*}+i+w+1} & \text { for Case III, } d \text { odd, } w \text { odd, } i \text { even, } \\
1 & \text { for Cases IA, IIA, IIC; or }
\end{array}
$$

Since $1<w<d$ it follows that $Y$ is convex precisely when $p(\Phi)$ satisfies one of the following: Case I, $s^{*}=0$; or Cases IA, IIA, IIC. Hence the result follows from (6.2).

The following is another important consequence of (4.3), (6.2), (A.3):

(6.4) Theorem. Given scalars $q, \alpha$ and $\beta$, if $\Gamma$ has classical parameters $(d, q, \alpha, \beta)$ (with standard $Q$-polynomial ordering) then $\Gamma_{Y}$ is distance-regular and has classical parameters $(w, q, \alpha, \beta)$. The converse also holds, provided $w \geqslant 3$. 
(6.5) Remark. In [31, Proposition 2], the author showed that $\iota\left(\Gamma_{Y}\right)$ is uniquely determined by $w$, and when $\Gamma$ is of "semilattice-type" the convexity of $Y$ was then derived from the existence of a specific example. We may remark that (6.3), (6.4) supersede these results and give an answer to the problem raised in [31, p. 907, Remark].

We end this section with a comment. Recall that $Y$ is called strongly closed if for any $x, y \in Y$ we have $\{z \in X: \partial(x, z)+\partial(z, y) \leqslant \partial(x, y)+1\} \subseteq Y$.

(6.6) With reference to (2.12), suppose $1<w<d$. Then $Y$ is strongly closed precisely when $\Gamma$ has classical parameters $(d, q, 0, \beta)$ (with standard $Q$-polynomial ordering).

Proof. We may assume that $Y$ is convex, or equivalently, by (6.3), that $\Gamma$ has classical parameters $(d, q, \alpha, \beta)$. In particular, $c_{i}(\Gamma)=c_{i}\left(\Gamma_{Y}\right)(1 \leqslant i \leqslant w)$. Note that $Y$ is then strongly closed if and only if $a_{i}(\Gamma)=a_{i}\left(\Gamma_{Y}\right)$ for all $1 \leqslant i \leqslant w$. Since $\Gamma_{Y}$ has classical parameters $(w, q, \alpha, \beta)$ by $(6.4)$, it follows from (2.5) that this happens precisely when $\alpha=0$, as desired.

\section{Quantum matroids and descendents}

There are many results on distance-regular graphs with the property that any pair of vertices $x, y$ is contained in a strongly closed subset with width $\partial(x, y)$; see e.g., [42, 19]. In view of the results of $\S 6$, in this section we assume $\Gamma$ has classical parameters and look at the implications of similar existence conditions on descendents.

First we recall some facts concerning quantum matroids [37] and related distanceregular graphs. A finite nonempty poset $^{9} \mathscr{P}$ is a quantum matroid if

(QM1) $\mathscr{P}$ is ranked;

(QM2) $\mathscr{P}$ is a (meet) semilattice;

(QM3) For all $x \in \mathscr{P}$, the interval $[0, x]$ is a modular atomic lattice;

(QM4) For all $x, y \in \mathscr{P}$ satisfying $\operatorname{rank}(x)<\operatorname{rank}(y)$, there is an atom $a \in \mathscr{P}$ such that $a \leqslant y, a \notin x$ and $x \vee a$ exists in $\mathscr{P}$.

We say $\mathscr{P}$ is nontrivial if $\mathscr{P}$ has rank $d \geqslant 2$ and is not a modular atomic lattice. Suppose $\mathscr{P}$ is nontrivial. Then $\mathscr{P}$ is called $q$-line regular if each rank 2 element covers exactly $q+1$ elements; $\mathscr{P}$ is $\beta$-dual-line regular if each element with rank $d-1$ is covered by exactly $\beta+1$ elements; $\mathscr{P}$ is $\alpha$-zig-zag regular if for all pairs $(x, y)$ such that $\operatorname{rank}(x)=$ $d-1, \operatorname{rank}(y)=d$ and $x$ covers $x \wedge y$, there are exactly $\alpha+1$ pairs $\left(x_{1}, y_{1}\right)$ such that $y_{1}$ covers both $x$ and $x_{1}$, and $y$ covers $x_{1}$. We say $\mathscr{P}$ is regular if $\mathscr{P}$ is line regular, dual-line regular and zig-zag regular. Suppose $\mathscr{P}$ is nontrivial and regular with parameters $(d, q, \alpha, \beta)$. Let $\operatorname{top}(\mathscr{P})$ be the top fiber of $\mathscr{P}$ and set $R=\{(x, y) \in \operatorname{top}(\mathscr{P}) \times \operatorname{top}(\mathscr{P}): x, y$ cover $x \wedge y\}$. Then by [37, Theorem 38.2], $\Gamma=(\operatorname{top}(\mathscr{P}), R)$ is distance-regular. Moreover, it has classical parameters $(d, q, \alpha, \beta)$ provided that the diameter equals $d$.

\footnotetext{
${ }^{9}$ See, e.g., [29, Chapter 3] for terminology from poset theory.
} 
We now list five examples of nontrivial regular quantum matroids from [37]. In (7.1)(7.5) below, the partial order on $\mathscr{P}$ will always be defined by inclusion and $Y$ will denote a nontrivial descendent of $\Gamma$.

(7.1) Example. The truncated Boolean algebra $B(d, \nu)(\nu>d)$. Let $\Omega$ be a set of size $\nu$ and $\mathscr{P}=\{x \subseteq \Omega:|x| \leqslant d\}$. $\mathscr{P}$ has parameters $(d, 1,1, \nu-d)$ and $\operatorname{top}(\mathscr{P})$ induces the Johnson graph $J(\nu, d)[3, \S 9.1]$. If $\nu \geqslant 2 d$ then $Y$ satisfies one of the following: (i) $Y=\{x \in \operatorname{top}(\mathscr{P}): u \subseteq x\}$ for some $u \subseteq \Omega$ with $|u|=w^{*}$; (ii) $\nu=2 d$ and $Y=\{x \in \operatorname{top}(\mathscr{P}): x \subseteq u\}$ for some $u \subseteq \Omega$ with $|u|=d+w$.

(7.2) Example. The Hamming matroid $H(d, \ell)(\ell \geqslant 2)$. Let $\Omega_{1}, \Omega_{2}, \ldots, \Omega_{d}$ be pairwise disjoint sets of size $\ell, \Omega=\bigcup_{i=1}^{d} \Omega_{i}$ and $\mathscr{P}=\left\{x \subseteq \Omega:\left|x \cap \Omega_{i}\right| \leqslant 1(1 \leqslant i \leqslant d)\right\}$. $\mathscr{P}$ has parameters $(d, 1,0, \ell-1)$ and $\operatorname{top}(\mathscr{P})$ induces the Hamming graph $H(d, \ell)[3, \S 9.2]$. $Y$ is of the form $\{x \in \operatorname{top}(\mathscr{P}): u \subseteq x\}$ for some $u \in \mathscr{P}$ with $|u|=w^{*}$.

(7.3) Example. The truncated projective geometry $L_{q}(d, \nu)(\nu>d)$. Let $\mathscr{P}$ be the set of subspaces $x$ of $\mathbb{F}_{q}^{\nu}$ with $\operatorname{dim} x \leqslant d$. $\mathscr{P}$ has parameters $(d, q, q, \beta)$ where $\beta+1=\left[\begin{array}{c}\nu-d+1 \\ 1\end{array}\right]_{q}$, and $\operatorname{top}(\mathscr{P})$ induces the Grassmann graph $J_{q}(\nu, d)[3, \S 9.3]$. If $\nu \geqslant 2 d$ then $Y$ satisfies one of the following: (i) $Y=\{x \in \operatorname{top}(\mathscr{P}): u \subseteq x\}$ for some subspace $u \subseteq \mathbb{F}_{q}^{\nu}$ with $\operatorname{dim} u=w^{*}$; (ii) $\nu=2 d$ and $Y=\{x \in \operatorname{top}(\mathscr{P}): x \subseteq u\}$ for some subspace $u \subseteq \mathbb{F}_{q}^{\nu}$ with $\operatorname{dim} u=d+w$.

(7.4) Example. The attenuated space $A_{q}(d, d+e)(e \geqslant 1)$. Fix a subspace $E$ of $\mathbb{F}_{q}^{d+e}$ with $\operatorname{dim} E=e$, and let $\mathscr{P}$ be the set of subspaces $x$ of $\mathbb{F}_{q}^{d+e}$ with $x \cap E=0$. $\mathscr{P}$ has parameters $\left(d, q, q-1, q^{e}-1\right)$, and $\operatorname{top}(\mathscr{P})$ induces the bilinear forms graph $\operatorname{Bil}_{q}(d, e)[3$, §9.5A]. If $d \leqslant e$ then $Y$ satisfies one of the following: (i) $Y=\{x \in \operatorname{top}(\mathscr{P}): u \subseteq x\}$ for some subspace $u \subseteq \mathbb{F}_{q}^{d+e}$ with $\operatorname{dim} u=w^{*}$ and $u \cap E=0$; (ii) $d=e$ and $Y=\{x \in$ $\operatorname{top}(\mathscr{P}): x \subseteq u\}$ for some subspace $u \subseteq \mathbb{F}_{q}^{d+e}$ with $\operatorname{dim} u=d+w$ and $\operatorname{dim} u \cap E=w$.

(7.5) Example. The classical polar spaces. Let $V$ be one of the following spaces over $\mathbb{F}_{q}$ equipped with a nondegenerate form:

\begin{tabular}{cclc}
\hline Name & $\operatorname{dim} V$ & \multicolumn{1}{c}{ Form } & $e$ \\
\hline$\left[C_{d}(q)\right]$ & $2 d$ & alternating & 1 \\
{$\left[B_{d}(q)\right]$} & $2 d+1$ & quadratic & 1 \\
{$\left[D_{d}(q)\right]$} & $2 d$ & quadratic (Witt index $d)$ & 0 \\
{$\left[{ }^{2} D_{d+1}(q)\right]$} & $2 d+2$ & quadratic (Witt index $d)$ & 2 \\
{$\left[{ }^{2} A_{2 d}(\ell)\right]$} & $2 d+1$ & Hermitean $\left(q=\ell^{2}\right)$ & $\frac{3}{2}$ \\
{$\left[{ }^{2} A_{2 d-1}(\ell)\right]$} & $2 d$ & Hermitean $\left(q=\ell^{2}\right)$ & $\frac{1}{2}$ \\
\hline
\end{tabular}

Let $\mathscr{P}$ be the set of isotropic subspaces of $V . \mathscr{P}$ has parameters $\left(d, q, 0, q^{e}\right)$ and top $(\mathscr{P})$ induces the dual polar graph on $V[3, \S 9.4]$. $Y$ is of the form $\{x \in \operatorname{top}(\mathscr{P}): u \subseteq x\}$ for some $u \in \mathscr{P}$ with $\operatorname{dim} u=w^{*}$.

We recall the following isomorphisms: $J(\nu, d) \cong J(\nu, \nu-d), J_{q}(\nu, d) \cong J_{q}(\nu, \nu-d)$, $\operatorname{Bil}_{q}(d, e) \cong \operatorname{Bil}_{q}(e, d)$. Note also that, in each of (7.1)-(7.5), the descendents with any fixed width form a single orbit under the full automorphism group of $\Gamma$. 
(7.6) Theorem([37, Theorem 39.6]). Every nontrivial regular quantum matroid with rank at least four is isomorphic to one of (7.1)-(7.5).

Now we return to the general situation (2.12). Let $\mathscr{P}$ be a nonempty family of descendents of $\Gamma$. We say $\mathscr{P}$ satisfies $(U D)_{i}$ if any two vertices $x, y \in X$ at distance $i$ are contained in a unique descendent in $\mathscr{P}$, denoted $Y(x, y)$, with width $i$. We shall assume the following three conditions until (7.20):

(7.7) $\Gamma$ has classical parameters $(d, q, \alpha, \beta)$;

(7.8) $\mathscr{P}$ satisfies $(\mathrm{UD})_{i}$ for all $i$;

(7.9) $Y_{1} \cap Y_{2} \in \mathscr{P}$ for all $Y_{1}, Y_{2} \in \mathscr{P}$ such that $Y_{1} \cap Y_{2} \neq \emptyset$.

Referring to $(7.7)-(7.9)$, define a partial order $\leqslant$ on $\mathscr{P}$ by reverse inclusion. Our goal is to show that $\mathscr{P}$ is a nontrivial regular quantum matroid. Note that $X$ is the minimal element of $\mathscr{P}$ and the maximal elements of $\mathscr{P}$ are precisely the singletons. We shall freely use (2.8), (4.4), (6.3), (6.4). In particular, note that every $Y \in \mathscr{P}$ is convex, $\Gamma_{Y}$ is distance-regular with classical parameters $(w(Y), q, \alpha, \beta)$, and $\mathscr{P}_{Y}:=\{Z \in \mathscr{P}: Z \subseteq Y\}$ is a family of descendents of $\Gamma_{Y}$.

(7.10) For $0 \leqslant i \leqslant j \leqslant d$, we have $|\{Y \in \mathscr{P}: x, y \in Y, w(Y)=j\}|=\left[\begin{array}{l}d-i \\ j-i\end{array}\right]_{q}$ for any two vertices $x, y \in X$ with $\partial(x, y)=i$. In particular, $q$ is a positive integer.

Proof. Count in two ways the sequences $\left(z_{1}, \ldots, z_{j-i}, Y\right)$ such that $z_{l} \in \Gamma_{i+l}(x) \cap \Gamma\left(z_{l-1}\right)$ $(1 \leqslant l \leqslant j-i)$ and $Y=Y\left(x, z_{j-i}\right)$, where $z_{0}=y$.

(7.11) $\mathscr{P}_{Y}$ satisfies $(U D)_{i}$ in $\Gamma_{Y}$ for all $Y \in \mathscr{P}$ and $i$.

Proof. Let $x, y \in Y$ and set $Z=Y(x, y) \in \mathscr{P}$. Then $Y \cap Z$ belongs to $\mathscr{P}_{Y}$ and contains $x$ and $y$, so that $Z=Y \cap Z \in \mathscr{P}_{Y}$.

(7.12) For $0 \leqslant i \leqslant j \leqslant d$, we have $|\{Z \in \mathscr{P}: Y \subseteq Z, w(Z)=j\}|=\left[\begin{array}{c}d-i \\ j-i\end{array}\right]_{q}$ for every $Y \in \mathscr{P}$ with $w(Y)=i$.

Proof. Pick $x, y \in Y$ with $\partial(x, y)=i$ and let $Z \in \mathscr{P}$. Then by (7.11) we find $x, y \in Z$ if and only if $Y \subseteq Z$. Hence the result follows from (7.10).

(7.13) भ satisfies (QM1) and $\operatorname{rank}(Y)=w^{*}(Y)$ for every $Y \in \mathscr{P}$.

Proof. Pick $Y, Z \in \mathscr{P}$ with $Y \subseteq Z$. Applying (7.12) to $\left(\Gamma_{Z}, \mathscr{P}_{Z}\right)$, we find $Y$ covers $Z$ precisely when $w(Z)=w(Y)+1$, or equivalently, $w^{*}(Z)=w^{*}(Y)-1$. Hence $\operatorname{rank}(Y)$ is well defined and equals $w^{*}(Y)$.

(7.14) $\mathscr{P}$ satisfies (QM2). Moreover, $w\left(Y_{1} \wedge Y_{2}\right)=w\left(Y_{1} \cup Y_{2}\right)$ for any $Y_{1}, Y_{2} \in \mathscr{P}$. 
Proof. Let $Y_{1}, Y_{2} \in \mathscr{P}$ and suppose $Y_{1} \nsubseteq Y_{2}, Y_{2} \nsubseteq Y_{1}$. Since $Y_{1}, Y_{2}$ are completely regular, for every $x \in X$ and $i \in\{1,2\}$ we have $w\left(\{x\} \cup Y_{i}\right)=\partial\left(x, Y_{i}\right)+w\left(Y_{i}\right)$. Hence there are $x_{1} \in Y_{1}, x_{2} \in Y_{2}$ such that $\partial\left(x_{1}, x_{2}\right)=w\left(Y_{1} \cup Y_{2}\right)$. Set $Z=Y\left(x_{1}, x_{2}\right)$. Pick $y_{1} \in Y_{1}$ with $\partial\left(x_{2}, y_{1}\right)=\partial\left(x_{2}, Y_{1}\right)=\partial\left(x_{1}, x_{2}\right)-w\left(Y_{1}\right)$. Then $\partial\left(x_{1}, y_{1}\right)=w\left(Y_{1}\right)$, so that $y_{1} \in Z$ and $Y_{1}=Y\left(x_{1}, y_{1}\right) \subseteq Z$ by (7.11). Likewise, $Y_{2} \subseteq Z$. Hence $Z$ is a lower bound for $Y_{1}, Y_{2}$. Any lower bound for $Y_{1}, Y_{2}$ contains $x_{1}, x_{2}$, and thus $Z$ by (7.11), whence $Z=Y_{1} \wedge Y_{2}$.

(7.15) भ satisfies (QM3).

Proof. Let $Y \in \mathscr{P}$. Then the interval $[X, Y]$ is a lattice since $\mathscr{P}$ satisfies (QM2) by (7.14). By (7.12), every $Z \in[X, Y]$ with $w^{*}(Z) \geqslant 2$ covers $\left[\begin{array}{c}w^{*}(Z) \\ 1\end{array}\right]_{q} \geqslant 2$ elements in $\mathscr{P}$, so that $[X, Y]$ is atomic. Let $Y_{1}, Y_{2} \in[X, Y]$ be distinct and suppose $w^{*}\left(Y_{1}\right)=w^{*}\left(Y_{2}\right)$. Set $i=w\left(Y_{1}\right), j=w^{*}\left(Y_{1}\right)$ for brevity. We claim $w^{*}\left(Y_{1} \wedge Y_{2}\right)=j-1$ if and only if $w^{*}\left(Y_{1} \cap Y_{2}\right)=j+1$, where we recall $Y_{1} \vee Y_{2}=Y_{1} \cap Y_{2}$. First suppose $w^{*}\left(Y_{1} \cap Y_{2}\right)=j+1$. Then $w\left(Y_{1} \cap Y_{2}\right)=i-1$ and by (4.4), (2.8) we find $Y_{1} \cap Y_{2}$ has covering radius one in each of $\Gamma_{Y_{1}}, \Gamma_{Y_{2}}$, so that $w\left(Y_{1} \cup Y_{2}\right) \leqslant w\left(Y_{1} \cap Y_{2}\right)+2=i+1$. Since $w\left(Y_{1} \wedge Y_{2}\right)>i$, we find $w\left(Y_{1} \wedge Y_{2}\right)=i+1$ by (7.14) and thus $w^{*}\left(Y_{1} \wedge Y_{2}\right)=j-1$. Next suppose $w^{*}\left(Y_{1} \wedge Y_{2}\right)=j-1$. Then by (7.12) and since $\left[\begin{array}{l}j \\ 1\end{array}\right]_{q}>\left[\begin{array}{c}j-1 \\ 1\end{array}\right]_{q}$, there is $C \in \mathscr{P}$ such that $w^{*}(C)=1, Y_{1} \subseteq C, Y_{1} \wedge Y_{2} \nsubseteq C$. We have $Y_{1}=C \cap\left(Y_{1} \wedge Y_{2}\right)$ and hence $Y_{1} \cap Y_{2}=C \cap Y_{2}$. Since $\hat{C} \circ \hat{Y}_{2} \in \sum_{l=0}^{j+1} E_{l} \mathbb{C}^{X}$ by virtue of (2.3), we find $w^{*}\left(Y_{1} \cap Y_{2}\right) \leqslant j+1$. By (2.8) and since $w\left(Y_{1} \cap Y_{2}\right)<i$, it follows that $w^{*}\left(Y_{1} \cap Y_{2}\right)=j+1$. The claim now follows, and therefore $[X, Y]$ is modular.

(7.16) भ satisfies (QM4).

Proof. Let $Y_{1}, Y_{2} \in \mathscr{P}$ and suppose $w^{*}\left(Y_{1}\right)<w^{*}\left(Y_{2}\right)$. First assume $Y_{1} \cap Y_{2} \neq \emptyset$. Then by (7.12) and since $\left[\begin{array}{c}w^{*}\left(Y_{2}\right) \\ 1\end{array}\right]_{q}>\left[\begin{array}{c}w^{*}\left(Y_{1}\right) \\ 1\end{array}\right]_{q}$, there is $C \in \mathscr{P}$ such that $w^{*}(C)=1, Y_{2} \subseteq C, Y_{1} \nsubseteq$ $C$. Since $Y_{1} \cap C \neq \emptyset$ we find $Y_{1} \vee C=Y_{1} \cap C$. Next assume $Y_{1} \cap Y_{2}=\emptyset$, and pick $x_{1}, y_{1} \in Y_{1}$ and $x_{2} \in Y_{2}$ such that $\partial\left(x_{1}, x_{2}\right)=w\left(Y_{1} \cup Y_{2}\right)$ and $\partial\left(x_{2}, y_{1}\right)=\partial\left(x_{2}, Y_{1}\right)=\partial\left(x_{1}, x_{2}\right)-w\left(Y_{1}\right)$ as in the proof of (7.14). Set $Z_{1}=Y_{1} \wedge Y_{2}, Z_{2}=\left\{y_{1}\right\} \wedge Y_{2}$. Then $Z_{2} \subseteq Z_{1}$, and since $w\left(\left\{y_{1}\right\} \cup Y_{2}\right)=\partial\left(y_{1}, Y_{2}\right)+w\left(Y_{2}\right) \leqslant \partial\left(y_{1}, x_{2}\right)+w\left(Y_{2}\right)=\partial\left(x_{1}, x_{2}\right)-w\left(Y_{1}\right)+w\left(Y_{2}\right)<$ $w\left(Y_{1} \cup Y_{2}\right)$, it follows from (7.14) that $w\left(Z_{1}\right)>w\left(Z_{2}\right)$, i.e., $w^{*}\left(Z_{1}\right)<w^{*}\left(Z_{2}\right)$. Again there is $C \in \mathscr{P}$ such that $w^{*}(C)=1, Z_{2} \subseteq C, Z_{1} \nsubseteq C$. Note that $Y_{2} \subseteq C$ and $Y_{1} \nsubseteq C$. Since $y_{1} \in C$, we find $Y_{1} \cap C \neq \emptyset$ and $Y_{1} \vee C=Y_{1} \cap C$, as desired.

(7.17) $\mathscr{P}$ is $q$-line regular, $\beta$-dual-line regular and $\alpha$-zig-zag regular.

Proof. By (7.12), $\mathscr{P}$ is $q$-line regular. Pick any $Y \in \mathscr{P}$ with $w(Y)=1$. Then $|Y|=\beta+1$ by $(6.4),(2.5)$, so that $\mathscr{P}$ is $\beta$-dual-line regular. Let $x \in X$ and suppose $w(\{x\} \wedge Y)=2$, i.e., $\partial(x, Y)=1$. We count the pairs $(y, Z) \in X \times \mathscr{P}$ such that $w(Z)=1, y \in Y$, $y \in Z, x \in Z$. Note that $x, y$ must be adjacent and $Z=Y(x, y)$. Hence the number of such pairs is $|\Gamma(x) \cap Y|$. By (6.4), (2.5), the strongly regular graph $\Delta=\Gamma_{\{x\} \wedge Y}$ satisfies $k(\Delta)=\beta(q+1), a_{1}(\Delta)=\beta+\alpha q-1, c_{2}(\Delta)=(\alpha+1)(q+1)$, and hence ([3, Theorem 1.3.1]) has smallest eigenvalue $\theta_{2}(\Delta)=-(q+1)$, so that $Y$ attains the Hoffman bound $1-k(\Delta) / \theta_{2}(\Delta)=\beta+1$. By [3, Proposition 1.3.2], $|\Gamma(x) \cap Y|=-c_{2}(\Delta) / \theta_{2}(\Delta)=\alpha+1$ and therefore $\mathscr{P}$ is $\alpha$-zig-zag regular. 
To summarize:

(7.18) Proposition. Suppose $\Gamma$ and $\mathscr{P}$ satisfy (7.7)-(7.9). Then $\mathscr{P}$ is a nontrivial regular quantum matroid with parameters $(d, q, \alpha, \beta)$.

Hence it follows from (7.6) that

(7.19) Theorem. Suppose $\Gamma$ and $\mathscr{P}$ satisfy (7.7)-(7.9), and suppose further $d \geqslant 4$. Then $\Gamma$ is either a Johnson, Hamming, Grassmann, bilinear forms, or dual polar graph.

One may wish to modify the conditions (7.7)-(7.9) so that we still obtain a characterization similar to (7.19). For example, it seems natural to assume that $\mathscr{P}$ is the set of all descendents of $\Gamma$. We now show that in this case (7.9) is redundant.

(7.20) Proposition. Suppose $\mathscr{P}$ is the set of descendents of $\Gamma$. Then (7.7), (7.8) together imply (7.9).

Proof. Note that (7.10) holds without change. We next show (7.11). Let $i$ be given. By induction, assume that $\mathscr{P}_{Z}$ satisfies $(\mathrm{UD})_{l}$ in $\Gamma_{Z}$ for all $Z \in \mathscr{P}$ and $l>i$. Let $Y \in \mathscr{P}$ and pick any $x, y \in Y$ with $\partial(x, y)=i$. Since $\mathscr{P}_{Y}$ satisfies $(\mathrm{UD})_{i+1}$ in $\Gamma_{Y}$, for any $z \in \Gamma_{i+1}(x) \cap \Gamma(y) \cap Y$ we have $x, y \in Y(x, z) \subseteq Y$, so that by replacing $Y$ with $Y(x, z)$ we may assume $w(Y)=i+1$. Since $\left[\begin{array}{c}d-i \\ 1\end{array}\right]_{q}>\left[\begin{array}{c}d-i-1 \\ 1\end{array}\right]_{q}$, it follows from (7.10) that there is $C \in \mathscr{P}$ with $x, y \in C, Y \nsubseteq C, w^{*}(C)=1$. Set $Z=Y \cap C$. Suppose $w(Z)=i+1$ and pick $u, v \in Z$ with $\partial(u, v)=i+1$. Then $Y=Y(u, v)$. But since $u, v \in C$ and $\mathscr{P}_{C}$ satisfies (UD) $)_{i+1}$, we would have $Y \subseteq C$, a contradiction. Hence

$w(Z)=i$. Moreover, $\hat{Z}=\hat{Y} \circ \hat{C} \in \sum_{l=0}^{d-i} E_{l} \mathbb{C}^{X}$ by virtue of $(2.3)$, whence $w^{*}(Z) \leqslant d-i$. By (2.8) it follows that $Z \in \mathscr{P}$ and therefore $Y(x, y)=Z \subseteq Y$. Hence $\mathscr{P}_{Y}$ satisfies $(\mathrm{UD})_{i}$ in $\Gamma_{Y}$. We have now shown (7.11). Finally, we show (7.9). Let $Y_{1}, Y_{2} \in \mathscr{P}$ and suppose $N:=Y_{1} \cap Y_{2} \neq \emptyset$. Pick $x, y \in N$ such that $\partial(x, y)=w(N)$. Then $Y(x, y) \subseteq N$ by (7.11). Since $Y(x, y)$ is completely regular and has width $w(N)$, for every $z \in N$ we have $w(N) \geqslant w(\{z\} \cup Y(x, y))=\partial(z, Y(x, y))+w(N)$, so that $z \in Y(x, y)$ and thus $N=Y(x, y) \in \mathscr{P}$, as desired.

(7.21) Remark. It should be noted that $J(2 d, d), J_{q}(2 d, d), \operatorname{Bil}_{q}(d, d)$ are the only examples among the graphs listed in (7.19) which do not possess the property that "the set of all descendents satisfies (UD) ${ }_{i}$ for all $i$." This property seems particularly strong, so that it is a reasonable guess that (7.7) would also be redundant.

\section{Classifications}

In $\S 7$, we focused on the 5 families of distance-regular graphs associated with short regular semilattices (or nontrivial regular quantum matroids). In this section, we shall extend the classification of descendents to all of the 15 known infinite families with classical parameters and with unbounded diameter. We shall freely use (2.8), (6.3), (6.4). 
(8.1) Throughout this section, $Y$ shall always denote a nontrivial descendent of $\Gamma$ with width $w$. Descriptions of some of the graphs below involve $n \in\{2 d, 2 d+1\}$, in which cases we use the following notation:

$$
m= \begin{cases}2 d-1 & \text { if } n=2 d, \\ 2 d+1 & \text { if } n=2 d+1\end{cases}
$$

\section{Doob graphs}

Let $d_{1}, d_{2}$ be positive integers, and let $\Gamma=\operatorname{Doob}\left(d_{1}, d_{2}\right):=\Gamma^{1} \times \Gamma^{2} \times \cdots \times \Gamma^{d_{1}+d_{2}}$ be a Doob graph $[3, \S 9.2 \mathrm{~B}]$, where $\Gamma^{1}, \ldots, \Gamma^{d_{1}}$ are copies of the Shrikhande graph $S$ on 16 vertices and $\Gamma^{d_{1}+1}, \ldots, \Gamma^{d_{1}+d_{2}}$ are copies of the complete graph $K_{4}$ on 4 vertices. $\Gamma$ has classical parameters $(d, 1,0,3)$, where $d=2 d_{1}+d_{2}$. In particular, $\iota(\Gamma)=\iota(H(d, 4))$.

$\Gamma_{Y}$ has classical parameters $(w, 1,0,3)$, so that $|Y|=4^{w}$. Observe that the convex subsets of $X$ are precisely the direct products $Y^{1} \times Y^{2} \times \cdots \times Y^{d_{1}+d_{2}}$ where $Y^{1}, \ldots, Y^{d_{1}}$ are convex subsets of $V S$ and $Y^{d_{1}+1}, \ldots, Y^{d_{1}+d_{2}}$ are nonempty subsets of $V K_{4}$ (cf. [23, Proposition 5.11]). Since $S$ has clique number three, it follows that

(8.2) Theorem. Every descendent of $\operatorname{Doob}\left(d_{1}, d_{2}\right)$ is of the form $Y=Y^{1} \times Y^{2} \times \cdots \times$ $Y^{d_{1}+d_{2}}$, where either $Y^{i}=V \Gamma^{i}$ or $\left|Y^{i}\right|=1$, for each $i\left(1 \leqslant i \leqslant d_{1}+d_{2}\right)$.

\section{Halved cubes}

Let $\Gamma=\frac{1}{2} H(n, 2)(n \in\{2 d, 2 d+1\})$ be a halved cube $[3, \S 9.2 \mathrm{D}]$ with vertex set $X=X_{n}^{\varepsilon}$, where $\varepsilon \in \mathbb{F}_{2}$ and $X_{n}^{0}$ (resp. $X_{n}^{1}$ ) is the set of even- (resp. odd-)weight vectors of $\mathbb{F}_{2}^{n}$. $\Gamma$ has classical parameters $(d, 1,2, m)$.

For every $x \in \mathbb{F}_{2}^{n}$ let wt $(x)$ be its (Hamming) weight. First suppose $w>1$. Fix $x, y \in Y$ with $\partial(x, y)=w$. For simplicity of notation, we assume $x=\left(x_{1}, t\right), y=\left(y_{1}, t\right)$, where $x_{1}, y_{1} \in X_{2 w}^{\varepsilon_{1}}, t \in X_{n-2 w}^{\varepsilon_{2}}\left(\varepsilon_{1}, \varepsilon_{2} \in \mathbb{F}_{2}, \varepsilon_{1}+\varepsilon_{2}=\varepsilon\right)$ and $\operatorname{wt}\left(x_{1}-y_{1}\right)=2 w$. Note that $X_{2 w}^{\varepsilon_{1}} \times\{t\} \subseteq Y$ by convexity. Let $z=\left(z_{1}, z_{2}\right) \in Y \backslash\left(X_{2 w}^{\varepsilon_{1}} \times\{t\}\right)$. Then it follows that $\operatorname{wt}\left(z_{2}-t\right)=1$. Moreover, for every $u=\left(u_{1}, u_{2}\right) \in Y \backslash\left(X_{2 w}^{\varepsilon_{1}} \times\{t\}\right)$ we have $u_{2}=z_{2}$, for otherwise by convexity and since $w>1$ there would be a vector $v=\left(v_{1}, v_{2}\right) \in Y$ such that $\operatorname{wt}\left(v_{2}-t\right)=2$. It follows that $\Gamma_{Y}$ has valency at most $\left(\begin{array}{c}2 w+1 \\ 2\end{array}\right)=w(2 w+1)$; but this is smaller than the expected valency $w m$ except when $n=2 d$ and $w=d-1$, in which case we must have $Y=\left(X_{2 w}^{\varepsilon_{1}} \times\{t\}\right) \cup\left(X_{2 w}^{1+\varepsilon_{1}} \times\left\{z_{2}\right\}\right)$.

Next suppose $w=1$. Then $|Y|=m+1$. On the other hand, $\frac{1}{2} H(n, 2)$ has maximal clique sizes 4 and $n$ [16, Theorem 14], from which it follows that $n=2 d$.

(8.3) Theorem. Let $Y$ be a nontrivial descendent of $\frac{1}{2} H(n, 2)$. Then $n=2 d$ and one of the following holds: (i) $w=1$ and $Y=\{x \in X$ : wt $(x-z)=1\}$ for some $z \in$ $X_{n}^{1+\varepsilon}=\mathbb{F}_{2}^{n} \backslash X$; (ii) $w=d-1$ and there are $a \in \mathbb{F}_{2}$ and $i \in\{1,2, \ldots, n\}$ such that $Y=\left\{x=\left(\xi_{1}, \xi_{2}, \ldots, \xi_{n}\right) \in X: \xi_{i}=a\right\}$. 


\section{Hemmeter graphs}

Let $q$ be an odd prime power and $\Sigma$ the dual polar graph on $\left[C_{d-1}(q)\right]$. The Hemmeter graph $\Gamma=\operatorname{Hem}_{d}(q)[3, \S 9.4 \mathrm{C}]$ is the extended bipartite double of $\Sigma$, so that $\Gamma$ has vertex set $X=X^{+} \cup X^{-}$, where $X^{ \pm}=\left\{x^{ \pm}: x \in V \Sigma\right\}$ are two copies of $V \Sigma$. $\Gamma$ has classical parameters $(d, q, 0,1)$, which coincide with those of the dual polar graph on $\left[D_{d}(q)\right]$.

If $w=1$ then $Y$ is an edge. Suppose $w>1$. Let $\stackrel{\circ}{Y}=\left\{x \in V \Sigma: x^{+} \in Y\right.$ or $\left.x^{-} \in Y\right\}$. Then $\stackrel{\circ}{Y}$ is a convex subset of $V \Sigma$ with width $\stackrel{\leftrightarrow}{w} \in\{w, w-1\}$. By [23, Proposition 5.19] and [16, Lemma 10], there is an isotropic subspace $u$ of $\left[C_{d-1}(q)\right]$ with $\operatorname{dim} u=d-1-\stackrel{\leftrightarrow}{w}$

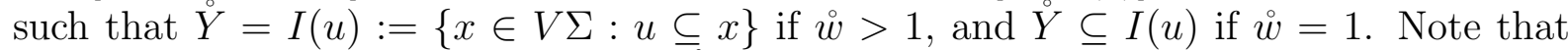
$|Y|=2 \prod_{i=1}^{w-1}\left(q^{i}+1\right)$ and $|I(u)|=\prod_{i=1}^{\stackrel{\leftrightarrow}{w}}\left(q^{i}+1\right)$ (cf. [3, Lemma 9.4.1]). If $\stackrel{\circ}{w}=w(>1)$ then $|Y|<|I(u)|=|\stackrel{\circ}{Y}|$, a contradiction. Hence $\stackrel{\circ}{w}=w-1$. It follows that

(8.4) Theorem. Let $Y$ be a nontrivial descendent of $\operatorname{Hem}_{d}(q)$. If $w=1$ then $Y$ is an edge. If $w>1$ then there is an isotropic subspace $u$ of $\left[C_{d-1}(q)\right]$ with $\operatorname{dim} u=w^{*}$ such that $Y=\left\{x^{+}, x^{-}: x \in V \Sigma, u \subseteq x\right\}$.

\section{Hermitean forms graphs}

In this subsection and the next, we realize $\Gamma$ as "affine subspaces" of dual polar graphs. See $[3, \S 9.5 \mathrm{E}]$ for the details.

Let $\ell$ be a prime power and let $\Delta$ be the dual polar graph on $\left[{ }^{2} A_{2 d-1}(\ell)\right]$. Fix a vertex $z$ of $\Delta$. The subgraph $\Gamma=\operatorname{Her}(d, \ell)$ of $\Delta$ induced on $X:=\Delta_{d}(z)$ is the Hermitean forms graph $[3, \S 9.5 \mathrm{C}] . \Gamma$ has classical parameters $\left(d,-\ell,-\ell-1,-(-\ell)^{d}-1\right)$.

By [23, Proposition 5.30], every noncomplete convex subset of $X$ is either of the form $I(u)=\{x \in X: u \subseteq x\}$ where $u$ is an isotropic subspace of $\left[{ }^{2} A_{2 d-1}(\ell)\right]$ with $\operatorname{dim} u \leqslant d-2$ and $z \cap u=0$, or isomorphic to the 4-cycle $K_{2,2}$. The latter case occurs only when $\ell=2$. Note that $I(u)$ induces $\operatorname{Her}(d-\operatorname{dim} u, \ell)$. By [16, Theorem 21], the maximal cliques of $\Gamma$ are the $I(u)$ with $\operatorname{dim} u=d-1$. Comparing the classical parameters it follows that

(8.5) Theorem. Her $(d, \ell)$ has no nontrivial descendent.

\section{Alternating forms graphs}

Let $\ell$ be a prime power and let $\Delta$ be the dual polar graph on $\left[D_{n}(\ell)\right]$, where $n \in\{2 d, 2 d+$ $1\}$. Fix a vertex $z$ of $\Delta$. The subgraph $\Gamma=\operatorname{Alt}(n, \ell)$ of the distance-2 graph of $\Delta$ induced on $X:=\Delta_{n}(z)$ is the alternating forms graph $[3, \S 9.5 \mathrm{~B}]$, i.e., $\Gamma=\Delta_{n}^{2}(z)$ with the notation of $\S 5$. $\Gamma$ has classical parameters $\left(d, \ell^{2}, \ell^{2}-1, \ell^{m}-1\right)$.

By [23, Proposition 5.26], every noncomplete convex subset of $X$ is of the form $I(u)=$ $\{x \in X: u \subseteq x\}$ where $u$ is an isotropic subspace of $\left[D_{n}(\ell)\right]$ with $\operatorname{dim} u \leqslant n-4$ and $z \cap u=0$. Note that $I(u)$ induces $\operatorname{Alt}(n-\operatorname{dim} u, \ell)$. By [16, Lemma 19], $\Gamma$ has clique number $\ell^{n-1}$ and the maximum cliques are of the form $C(x, v)=\{x\} \cup\{y \in \Gamma(x): x \cap y \subseteq$ $v$ \} where $x \in X$ and $v$ is a subspace of $x$ with $\operatorname{dim} v=n-1$. It follows that 
(8.6) Theorem. Let $Y$ be a nontrivial descendent of $\operatorname{Alt}(n, \ell)$. Then $n=2 d$ and $Y$ takes one of the following forms: (i) $w=1$ and $Y=C(x, v)$; (ii) $w=d-1$ and $Y=I(u)$ with $\operatorname{dim} u=1$.

\section{Quadratic forms graphs}

Let $\ell$ be a prime power and let $X$ be the set of quadratic forms on $V=\mathbb{F}_{\ell}^{n-1}$ over $\mathbb{F}_{\ell}$, where $n \in\{2 d, 2 d+1\}$. For $x \in X$ let $\operatorname{Rad} x=x^{-1}(0) \cap \operatorname{Rad} B_{x}$ and $\operatorname{rk} x=\operatorname{dim}(V / \operatorname{Rad} x)$, where $B_{x}$ is the symmetric bilinear form associated with $x$ and $\operatorname{Rad} B_{x}$ denotes its radical. Let $\Gamma=\operatorname{Quad}(n-1, \ell)$ be the quadratic forms graph on $V[3, \S 9.6]: x, y \in X$ are adjacent if $\operatorname{rk}(x-y)=1$ or 2 . $\Gamma$ has classical parameters $\left(d, \ell^{2}, \ell^{2}-1, \ell^{m}-1\right)$. We remark that if $\ell$ is odd then $\Gamma$ is isomorphic to the subgraph of the distance 1-or-2 graph of the dual polar graph $\Sigma$ on $\left[C_{n-1}(\ell)\right]$ induced on $\Sigma_{n-1}(z)$ with $z \in V \Sigma$, or equivalently, $\Gamma$ is isomorphic to $\Delta_{n}^{2}(z)$, where $\Delta=\operatorname{Hem}_{n}(\ell)$ and $z \in V \Delta$; see [9].

By [23, Proposition 5.36], [26, Theorem 1.2], every noncomplete convex subset of $X$ is of the form ${ }^{10} I(x, u)=\{y \in X: \operatorname{Rad}(x-y) \supseteq u\}$ where $x \in X$ and $u$ is a subspace of $V$ with $\operatorname{dim} u \leqslant n-4$. Note that $I(x, u)$ induces $\operatorname{Quad}(n-1-\operatorname{dim} u, \ell)$. By [17, Theorems $14,24,26], \Gamma$ has clique number $\ell^{n-1}$ and there are two types of maximum cliques: A type 1 clique is defined to be $C(x)=\{y \in X: \operatorname{rk}(x-y) \leqslant 1\}$ where $x \in X$. If $q$ is odd then a type 2 clique is defined to be $C(x, u)=\left\{y \in X:\left.B_{y}\right|_{u \times u}=\left.B_{x}\right|_{u \times u}\right\}$ where $x \in X$ and $u$ is a subspace of $V$ with $\operatorname{dim} u=n-2$. If $q$ is even then it is defined to be $C(x, u, \gamma, a)=\left\{y \in X:\left.B_{y}\right|_{u \times u}=\left.B_{x}\right|_{u \times u},(x-y)(\xi)=a\left(\left(B_{x}-B_{y}\right)(\gamma, \xi)\right)^{2}\right.$ for all $\left.\xi \in u\right\}$ where $x \in X, u$ a subspace of $V$ with $\operatorname{dim} u=n-2, \gamma \in V \backslash u$ and $a \in \mathbb{F}_{\ell}$. See also [6, 18]. Cliques of types 1 and 2 are called grand cliques. It follows that

(8.7) Theorem. Let $Y$ be a nontrivial descendent of $\operatorname{Quad}(n-1, \ell)$. Then $n=2 d$ and $Y$ takes one of the following forms: (i) $w=1$ and $Y$ is a grand clique; (ii) $w=d-1$ and $Y=I(x, u)$ with $\operatorname{dim} u=1$.

\section{Unitary dual polar graphs with second $Q$-polynomial ordering}

Let $\ell$ be a prime power and let $\Gamma=U(2 d, \ell)$ be the dual polar graph on $\left[{ }^{2} A_{2 d-1}(\ell)\right]$. Besides the ordinary $\left(d, \ell^{2}, 0, \ell\right), \Gamma$ has another set of classical parameters $(d,-\ell, \alpha, \beta)$,

where $\alpha+1=\frac{1+\ell^{2}}{1-\ell}$ and $\beta+1=\frac{1-(-\ell)^{d+1}}{1-\ell}$ [3, Corollary 6.2.2]. Here we consider the standard $Q$-polynomial ordering with respect to the latter classical parameters; it is $\left\{E_{0}, E_{d}, E_{1}, E_{d-1}, \ldots\right\}$ in terms of the ordinary ordering $\left\{E_{i}\right\}_{i=0}^{d}$.

By [23, Proposition 5.19], every noncomplete convex subset of $X$ is of the form $I(u)=$ $\{x \in X: u \subseteq x\}$ for some isotropic subspace $u$ of $\left[{ }^{2} A_{2 d-1}(\ell)\right]$ with $\operatorname{dim} u=w^{*}$. By [16, Lemma 10], the maximal cliques of $\Gamma$ are the $I(u)$ with $\operatorname{dim} u=d-1$. It follows that

(8.8) Theorem. $U(2 d, \ell)$ (with second $Q$-polynomial ordering) has no nontrivial descendent.

\footnotetext{
${ }^{10}$ If $\ell$ is odd, then in terms of the above identification $X=\Sigma_{n-1}(z)$, every noncomplete convex subgraph of $\Gamma$ is rewritten as $I(u)=\{y \in X: u \subseteq y\}$ for some isotropic subspace of $\left[C_{n-1}(\ell)\right]$ with $\operatorname{dim} u \leqslant n-4$ and $z \cap u=0$.
} 


\section{Half dual polar graphs}

Let $\ell$ be a prime power and let $\Delta$ be the bipartite dual polar graph on $\left[D_{n}(\ell)\right]$ with $V \Delta=X^{+} \cup X^{-}$, where $n \in\{2 d, 2 d+1\}$ and $X^{+}, X^{-}$are bipartite halves of $\Delta$. The pathlength distance for $\Delta$ is denoted $\partial_{\Delta}$. Let $\Gamma=D_{n, n}(\ell)$ be a halved graph of $\Delta$ with vertex set $X=X^{\varepsilon}$ where $\varepsilon \in\{+,-\}$, whence $2 \partial=\left.\partial_{\Delta}\right|_{X \times X}[3, \S 9.4 \mathrm{C}]$. $\Gamma$ has classical parameters $\left(d, \ell^{2}, \alpha, \beta\right)$, where $\alpha+1=\left[\begin{array}{l}3 \\ 1\end{array}\right]_{\ell}$ and $\beta+1=\left[\begin{array}{c}m+1 \\ 1\end{array}\right]_{\ell}$. Pepe, Storme and Vanhove $[28, \S 5]$ recently classified the descendents of $\Gamma$ when $n=2 d$ and $w=d-1$, partly based on the Erdős-Ko-Rado theorem for Grassmann graphs (cf. [31, Theorem 3]). Our approach below uses (8.6) instead.

First suppose $w>1$. Fix $x \in Y$ and pick any $z \in \Delta_{n-2 w}(x)$ such that $\Delta_{n}(z) \cap Y \neq \emptyset$. By (5.2), (8.6) we find $n=2 d, w=d-1$ and there is an isotropic subspace $u$ of $\left[D_{2 d}(\ell)\right]$ with $\operatorname{dim} u=1$ and $z \cap u=0$ such that $Y_{0}:=\Delta_{2 d}(z) \cap Y=\left\{y \in \Delta_{2 d}(z): u \subseteq y\right\}$. Note that $z \in \Gamma(x) \backslash Y$ and $Y_{0}=\Gamma_{d}(z) \cap Y \subseteq\left(\Gamma_{Y}\right)_{d-1}(x)$. For convenience, let $\Xi$ be the dual polar graph on the residual polar space of $u$, so that $V \Xi=\{y \in V \Delta: u \subseteq y\}$.

(8.9) $x \in V \Xi$.

Proof. Suppose $u \nsubseteq x$. Let $x^{\dagger}=u+\left(x \cap u^{\perp}\right), z^{\dagger}=u+\left(z \cap u^{\perp}\right) \in V \Xi$. Then $\partial_{\Delta}(x, y)=$ $\partial_{\Delta}\left(x^{\dagger}, y\right)+1$ for any $y \in V \Xi$, and similarly for $z^{\dagger}$. Since $Y_{0} \subseteq \Xi_{2 d-3}\left(x^{\dagger}\right)$ we find $x^{\dagger} \in \Delta_{3}(z)$, i.e., $x^{\dagger} \in \Xi_{2}\left(z^{\dagger}\right)$. But then $\Gamma_{d}(x) \cap Y_{0}=\Xi_{2 d-1}\left(x^{\dagger}\right) \cap \Xi_{2 d-1}\left(z^{\dagger}\right) \neq \emptyset$, a contradiction.

(8.10) $\left(\Gamma_{Y}\right)_{d-1}(x) \subseteq V \Xi$.

Proof. Let $z_{1} \in \Gamma(x) \backslash Y$. Since $Y$ is completely regular in $\Gamma, Y_{1}:=\Gamma_{d}\left(z_{1}\right) \cap Y \neq \emptyset$. Let $u_{1}$ be the isotropic subspace of $\left[D_{2 d}(\ell)\right]$ with $\operatorname{dim} u_{1}=1, z_{1} \cap u_{1}=0$ and $Y_{1}=\left\{y \in \Gamma_{d}\left(z_{1}\right)\right.$ : $\left.u_{1} \subseteq y\right\}$. Note that $\left|Y_{0}\right|=\left|Y_{1}\right|=\ell^{(d-1)(2 d-1)}$ and $\left|\left(\Gamma_{Y}\right)_{d-1}(x)\right|=\prod_{i=1}^{d-1}\left(b_{i-1}\left(\Gamma_{Y}\right) / c_{i}\left(\Gamma_{Y}\right)\right)=$ $\ell^{(d-1)(2 d-3)}\left[\begin{array}{c}2 d-1 \\ 1\end{array}\right]_{\ell}$. Suppose $u \neq u_{1}$. Then $u+u_{1} \subseteq x$ by (8.9), so that by looking at the residual polar space of $u+u_{1}$ we find $\left|Y_{0} \cap Y_{1}\right| \leqslant|\operatorname{Alt}(2 d-2, \ell)|=\ell^{(d-1)(2 d-3)}$. If $\ell>2$ then $\left|Y_{0} \cap Y_{1}\right| \geqslant\left|Y_{0}\right|+\left|Y_{1}\right|-\left|\left(\Gamma_{Y}\right)_{d-1}(x)\right|>\ell^{(d-1)(2 d-3)}$, a contradiction. Hence $\ell=2$, $Y_{0} \cup Y_{1}=\left(\Gamma_{Y}\right)_{d-1}(x)$ and $Y_{0} \cap Y_{1}=\left\{y \in\left(\Gamma_{Y}\right)_{d-1}(x): u+u_{1} \subseteq y\right\}$, from which it follows that $Y_{0}=\left\{y \in\left(\Gamma_{Y}\right)_{d-1}(x): u \subseteq y\right\}, Y_{1}=\left\{y \in\left(\Gamma_{Y}\right)_{d-1}(x): u_{1} \subseteq y\right\}$. Pick $v \in Y_{0} \cap Y_{1}$. Since $a_{d-1}(\Gamma)>a_{d-1}\left(\Gamma_{Y}\right)$ there is $z_{2} \in \Gamma(x) \cap \Gamma_{d-1}(v) \backslash Y$. Let $u_{2}$ be the isotropic subspace of $\left[D_{2 d}(2)\right]$ with $\operatorname{dim} u_{2}=1, z_{2} \cap u_{2}=0$ and $Y_{2}:=\Gamma_{d}\left(z_{2}\right) \cap Y=\left\{y \in \Gamma_{d}\left(z_{2}\right): u_{2} \subseteq y\right\}$. Since $v \notin Y_{2}$ we find $Y_{2} \neq Y_{0}, Y_{1}$, so that $u_{2} \neq u, u_{1}$, and thus $\left|Y_{0} \cap Y_{2}\right|,\left|Y_{1} \cap Y_{2}\right| \leqslant 2^{(d-1)(2 d-3)}$ by the above argument. But $\max \left\{\left|Y_{0} \cap Y_{2}\right|,\left|Y_{1} \cap Y_{2}\right|\right\} \geqslant \frac{1}{2}\left|Y_{2}\right|>2^{(d-1)(2 d-3)}$, a contradiction. Hence $u=u_{1}$. Since $\left(\Gamma_{Y}\right)_{d-1}(x)=\bigcup_{z_{1} \in \Gamma(x) \backslash Y} \Gamma_{d}\left(z_{1}\right) \cap Y$, the proof is complete.

It follows from (8.9), (8.10) that $Y \subseteq V \Xi \cap X$, so that $\Gamma_{Y}$ is a subgraph of a halved graph of $\Xi$. Since $\iota\left(\Gamma_{Y}\right)=\iota\left(D_{2 d-1,2 d-1}(\ell)\right)$ we conclude $Y=V \Xi \cap X$.

Next suppose $w=1$. Then $|Y|=\left[\begin{array}{c}m+1 \\ 1\end{array}\right]_{\ell}$. By [16, Lemma 12] or [5, Theorem 3.5], $\Gamma$ has clique number $\left[\begin{array}{c}2 d \\ 1\end{array}\right]_{\ell}$ and the maximum cliques are of the form $\Delta(z)$ where $z \in V \Delta \backslash X$.

(8.11) Theorem. Let $Y$ be a nontrivial descendent of $D_{n, n}(\ell)$. Then $n=2 d$ and one of the following holds: (i) $w=1$ and $Y=\Delta(z)$ for some $z \in V \Delta \backslash X$; (ii) $w=d-1$ and $Y=\{x \in X: u \subseteq x\}$ for some isotropic subspace $u$ of $\left[D_{2 d}(\ell)\right]$ with $\operatorname{dim} u=1$. 


\section{Ustimenko graphs}

Let $\ell$ be an odd prime power and let $\Sigma$ be the dual polar graph on $\left[C_{n-1}(\ell)\right]$ with vertex set $X=V \Sigma$, where $n \in\{2 d, 2 d+1\}$. The path-length distance for $\Sigma$ is denoted $\partial_{\Sigma}$. The Ustimenko graph $\Gamma=$ Ust $_{n-1}(\ell)$ is the distance 1-or-2 graph of $\Sigma$, whence $\partial=\left\lceil\frac{1}{2} \partial_{\Sigma}\right\rceil[3$, $\S 9.4 \mathrm{C}] . \Gamma$ has classical parameters $\left(d, \ell^{2}, \alpha, \beta\right)$, where $\alpha+1=\left[\begin{array}{l}3 \\ 1\end{array}\right]_{\ell}$ and $\beta+1=\left[\begin{array}{c}m+1 \\ 1\end{array}\right]_{\ell}$. Note that $\Gamma$ may also be viewed as a halved graph of $\Delta=\operatorname{Hem}_{n}(\ell)$. Pepe et al. $[28, \S 7]$ classified the descendents of $\Gamma$ when $n=2 d$ and $w=d-1$ by a different approach from the following.

First suppose $w>1$. Fix $x \in Y$ and pick any $z \in \Delta_{n-2 w}(x)$ such that $\Delta_{n}(z) \cap Y \neq \emptyset$. By (5.2), (8.7) we find $n=2 d, w=d-1$ and there is an isotropic subspace $u$ of $\left[C_{2 d-1}(\ell)\right]$ with $\operatorname{dim} u=1$ and $z \cap u=0$ such that $Y_{0}:=\Delta_{2 d}(z) \cap Y=\left\{y \in \Delta_{2 d}(z): u \subseteq y\right\}$. ${ }^{11}$ Note that $z \in \Gamma(x) \backslash Y$ and $Y_{0}=\Gamma_{d}(z) \cap Y=\Sigma_{2 d-1}(z) \cap Y \subseteq\left(\Gamma_{Y}\right)_{d-1}(x)$. Let $\Xi$ be the dual polar graph on the residual polar space of $u$, so that $V \Xi=\{y \in V \Sigma: u \subseteq y\}$.

(8.12) $x \in V \Xi$.

Proof. Suppose $u \not \subset x$. Let $x^{\dagger}=u+\left(x \cap u^{\perp}\right), z^{\dagger}=u+\left(z \cap u^{\perp}\right) \in V \Xi$. Then $\Gamma_{d}(x) \cap Y_{0}=$ $\Xi_{2 d-2}\left(x^{\dagger}\right) \cap \Xi_{2 d-2}\left(z^{\dagger}\right) \neq \emptyset$, a contradiction.

(8.13) $\left(\Gamma_{Y}\right)_{d-1}(x) \subseteq V \Xi$.

Proof. Let $z_{1} \in \Gamma(x) \backslash Y$. Since $Y$ is completely regular in $\Gamma, Y_{1}:=\Gamma_{d}\left(z_{1}\right) \cap Y \neq \emptyset$. Let $u_{1}$ be the isotropic subspace of $\left[C_{2 d-1}(\ell)\right]$ with $\operatorname{dim} u_{1}=1, z_{1} \cap u_{1}=0$ and $Y_{1}=\{y \in$ $\left.\Gamma_{d}\left(z_{1}\right): u_{1} \subseteq y\right\}$. Note that $\left|Y_{0}\right|=\left|Y_{1}\right|=\ell^{(d-1)(2 d-1)}$ and $\left|\left(\Gamma_{Y}\right)_{d-1}(x)\right|=\ell^{(d-1)(2 d-3)}\left[\begin{array}{c}2 d-1 \\ 1\end{array} \ell^{\text {. }}\right.$ Suppose $u \neq u_{1}$. Then $u+u_{1} \subseteq x$ by (8.12), so that $\left|Y_{0} \cap Y_{1}\right| \leqslant|\operatorname{Quad}(2 d-3, \ell)|=$ $\ell^{(d-1)(2 d-3)}$. But $\left|Y_{0} \cap Y_{1}\right| \geqslant\left|Y_{0}\right|+\left|Y_{1}\right|-\left|\left(\Gamma_{Y}\right)_{d-1}(x)\right|>\ell^{(d-1)(2 d-3)}$, a contradiction. Hence $u=u_{1}$. Since $\left(\Gamma_{Y}\right)_{d-1}(x)=\bigcup_{z_{1} \in \Gamma(x) \backslash Y} \Gamma_{d}\left(z_{1}\right) \cap Y$, the proof is complete.

(8.14) $Y \subseteq V \Xi$

Proof. Suppose there is $y \in Y \backslash V \Xi$. Pick $v \in\left(\Gamma_{Y}\right)_{d-1}(x)$ such that $\partial(x, y)+\partial(y, v)=d-1$. Set $y^{\dagger}=u+\left(y \cap u^{\perp}\right) \in V \Xi$. Then by (8.12), (8.13) it follows that $\partial_{\Sigma}(x, v) \leqslant \partial_{\Sigma}\left(x, y^{\dagger}\right)+$ $\partial_{\Sigma}\left(y^{\dagger}, v\right)=\partial_{\Sigma}(x, y)+\partial_{\Sigma}(y, v)-2 \leqslant 2 d-4$, a contradiction.

By $(8.14)$ and since $\iota\left(\Gamma_{Y}\right)=\iota\left(\operatorname{Ust}_{2 d-2}(\ell)\right)$ we conclude $Y=V \Xi$.

Next suppose $w=1$. Then $|Y|=\left[\begin{array}{c}m+1 \\ 1\end{array}\right]_{\ell}$. By [5, Theorem 3.7], $\Gamma$ has clique number $\left[\begin{array}{c}2 d \\ 1\end{array}\right]_{\ell}$ and the maximum cliques are of the form $\Sigma(x) \cup\{x\}$ where $x \in X$.

(8.15) Theorem. Let $Y$ be a nontrivial descendent of $\operatorname{Ust}_{n-1}(\ell)$. Then $n=2 d$ and one of the following holds: (i) $w=1$ and $Y=\Sigma(x) \cup\{x\}$ for some $x \in X$; (ii) $w=d-1$ and $Y=\{x \in X: u \subseteq x\}$ for some isotropic subspace $u$ of $\left[C_{2 d-1}(\ell)\right]$ with $\operatorname{dim} u=1$.

\footnotetext{
${ }^{11}$ See footnote 10.
} 


\section{Twisted Grassmann graphs}

Let $q$ be a prime power and fix a hyperplane $H$ of $\mathbb{F}_{q}^{2 d+1}$. Let $X_{1}$ be the set of $(d+1)$ dimensional subspaces of $\mathbb{F}_{q}^{2 d+1}$ not contained in $H$, and $X_{2}$ the set of $(d-1)$-dimensional subspaces of $H$. The twisted Grassmann graph $\Gamma=\tilde{J}_{q}(2 d+1, d)[12,14,1,27]$ has vertex set $X=X_{1} \cup X_{2}$, and $x, y \in X$ are adjacent if $\operatorname{dim} x+\operatorname{dim} y-2 \operatorname{dim} x \cap y=2$. $\Gamma$ has classical parameters $(d, q, q, \beta)$, where $\beta+1=\left[\begin{array}{c}d+2 \\ 1\end{array}\right]_{q}$. Note that $X_{2}$ is a descendent of $\Gamma$ and induces $J_{q}(2 d, d-1)$. We observe

(8.16) $2 \partial(x, y)=\operatorname{dim} x+\operatorname{dim} y-2 \operatorname{dim} x \cap y \quad(x, y \in X)$.

(8.17) For $x, y, z \in X$, we have $\partial(x, z)+\partial(z, y)=\partial(x, y)$ if and only if $x \cap y \subseteq z=$ $(x \cap z)+(y \cap z)$.

Proof. Observe $\operatorname{dim} x \cap z+\operatorname{dim} y \cap z \leqslant \operatorname{dim} z+\operatorname{dim} x \cap y \cap z \leqslant \operatorname{dim} z+\operatorname{dim} x \cap y$, and equality holds if and only if $x \cap y \subseteq z=(x \cap z)+(y \cap z)$. Hence the result follows from (8.16).

(8.18) Let $Z$ be a nonempty subset of $X_{1}$ such that $\left\{z \in X_{1}: x \cap y \subseteq z=(x \cap z)+(y \cap z)\right\} \subseteq$ $Z$ for all $x, y \in Z$. Then at least one of the following holds: (i) there is a subspace $u$ of $\mathbb{F}_{q}^{2 d+1}$ with $\operatorname{dim} u=d-w(Z)+1$ such that $u \subseteq z$ for all $z \in Z$; (ii) there is a subspace $v$ of $\mathbb{F}_{q}^{2 d+1}$ with $\operatorname{dim} v=d+w(Z)+1$ and not contained in $H$ such that $z \subseteq v$ for all $z \in Z$.

Proof. Fix $x, y \in Z$ with $\partial(x, y)=w(Z)$ and recall $\operatorname{dim} x \cap y=d-w(Z)+1$ by (8.16). Let $z \in X_{1}$. We claim that if $x \cap y \nsubseteq z \nsubseteq x+y$ then $z \notin Z$. Suppose $z \in Z$. Let $\gamma \in(x \cap y) \backslash z$ and $\sigma \in z \backslash((x+y) \cup H)$. Let $E$ be a complementary subspace of $\langle\gamma\rangle$ in $x$ such that $x \cap z \subseteq E$. Set $z^{\dagger}=E+\langle\sigma\rangle \in X_{1}$. Since $x \cap z \subseteq z^{\dagger}=\left(x \cap z^{\dagger}\right)+\left(z \cap z^{\dagger}\right)$ we find $z^{\dagger} \in Z$. But then $\gamma \notin z^{\dagger} \cap y(=E \cap y) \subseteq x \cap y$ implies $\operatorname{dim} z^{\dagger} \cap y<d-w(Z)+1$ and thus $\partial\left(z^{\dagger}, y\right)>w(Z)$ by (8.16), a contradiction. Hence the claim follows. It follows that every $z \in Z$ satisfies $x \cap y \subseteq z$ or $z \subseteq x+y$ (or both). Next we claim that there is no pair $\left(z_{1}, z_{2}\right)$ of elements of $Z$ such that $x \cap y \nsubseteq z_{1} \subseteq x+y$ and $x \cap y \subseteq z_{2} \nsubseteq x+y$. Suppose such a pair $\left(z_{1}, z_{2}\right)$ exists. Let $\zeta \in z_{2} \backslash((x+y) \cup H)$. Let $E$ be a hyperplane of $z_{1}$ such that $z_{1} \cap z_{2} \subseteq E$. Set $z^{\ddagger}=E+\langle\zeta\rangle \in X_{1}$. Since $z_{1} \cap z_{2} \subseteq z^{\ddagger}=\left(z_{1} \cap z^{\ddagger}\right)+\left(z_{2} \cap z^{\ddagger}\right)$ we find $z^{\ddagger} \in Z$. But this is absurd since $x \cap y \nsubseteq z^{\ddagger} \nsubseteq x+y$. Hence the claim follows. Setting $u=x \cap y$ and $v=x+y$, we find that at least one of (i), (ii) holds.

(8.19) Let $Z$ be a nonempty convex subset of $X$. If there are vertices $x \in Z \cap X_{1}, y \in Z \cap X_{2}$ with $\partial(x, y)=w(Z)$, then for each $i=1,2$ at least one of the following holds: (i) there is a subspace $u$ of $H$ with $\operatorname{dim} u=d-w(Z)$ such that $u \subseteq z$ for all $z \in Z \cap X_{i}$; (ii) there is a subspace $v$ of $\mathbb{F}_{q}^{2 d+1}$ with $\operatorname{dim} v=d+w(Z)$ and not contained in $H$ such that $z \subseteq v$ for all $z \in Z \cap X_{i}$.

Proof. Similar to the proof of (8.18), by virtue of (8.17).

(8.20) Theorem. Let $Y$ be a nontrivial descendent of $\tilde{J}_{q}(2 d+1, d)$. Then $Y=\left\{x \in X_{2}\right.$ : $u \subseteq x\}$ for some subspace $u$ of $H$ with $\operatorname{dim} u=d-w-1$. 
Proof. Note that $\iota\left(\Gamma_{Y}\right)=\iota\left(J_{q}(d+w+1, w)\right)$, whence $|Y|=\left[\begin{array}{c}d+w+1 \\ w\end{array}\right]_{q}$. First suppose $Y \subseteq X_{1}$. Then in view of (8.17), if (8.18)(i) holds then $|Y| \leqslant\left[\begin{array}{c}d+w \\ w\end{array}\right]_{q}<\left[\begin{array}{c}d+w+1 \\ w\end{array}\right]_{q}$, and if (8.18)(ii) holds then $|Y| \leqslant\left[\begin{array}{c}d+w+1 \\ d+1\end{array}\right]_{q}-\left[\begin{array}{c}d+w \\ d+1\end{array}\right]_{q}<\left[\begin{array}{c}d+w+1 \\ w\end{array}\right]_{q}$, a contradiction. Hence $Y \nsubseteq X_{1}$. Next suppose $Y \cap X_{1}, Y \cap X_{2}$ are nonempty. Let $z \in Y \cap X_{1}, y \in Y \cap X_{2}$, and pick any $x \in Y$ with $\partial(x, y)=w, \partial(x, z)=w-\partial(z, y)$. Since $X_{2}$ is convex we find $x \in Y \cap X_{1}$. Let $w_{1}:=w\left(Y \cap X_{1}\right) \leqslant w$. By (8.17), $Y \cap X_{1}$ satisfies the assumptions of (8.18). If $Y \cap X_{1}$ satisfies (8.18)(i) then $\left|Y \cap X_{1}\right| \leqslant\left[\begin{array}{c}d+w_{1} \\ w_{1}\end{array}\right]_{q} \leqslant\left[\begin{array}{c}d+w \\ w\end{array}\right]_{q}$. If $Y \cap X_{1}$ satisfies (8.19)(ii) then $\left|Y \cap X_{1}\right| \leqslant\left[\begin{array}{c}d+w \\ d+1\end{array}\right]_{q} \leqslant\left[\begin{array}{c}d+w \\ w\end{array}\right]_{q}$. If $Y \cap X_{1}$ satisfies both (8.18)(ii) and (8.19)(i) then $\left|Y \cap X_{1}\right| \leqslant\left[\begin{array}{c}w+w_{1}+1 \\ w+1\end{array}\right]_{q} \leqslant\left[\begin{array}{c}d+w \\ w\end{array}\right]_{q}$ since $w<d$. Hence we always have $\left|Y \cap X_{1}\right| \leqslant\left[\begin{array}{c}d+w \\ w\end{array}\right]_{q}$. On the other hand, if $Y \cap X_{2}$ satisfies (8.19)(i) then $\left|Y \cap X_{2}\right| \leqslant\left[\begin{array}{c}d+w \\ w-1\end{array}\right]_{q}$, and if $Y \cap X_{2}$ satisfies (8.19)(ii) then $\left|Y \cap X_{2}\right| \leqslant\left[\begin{array}{c}d+w-1 \\ w\end{array}\right]_{q}$. Since $q^{d+1}\left[\begin{array}{c}d+w \\ w-1\end{array}\right]_{q}>\left[\begin{array}{c}d+w-1 \\ w\end{array}\right]_{q}$, it follows that $|Y|=\left|Y \cap X_{1}\right|+\left|Y \cap X_{2}\right|<\left[\begin{array}{c}d+w \\ w\end{array}\right]_{q}+q^{d+1}\left[\begin{array}{c}d+w \\ w-1\end{array}\right]_{q}=\left[\begin{array}{c}d+w+1 \\ w\end{array}\right]_{q}$, a contradiction. Hence $Y \subseteq X_{2}$, and by (4.4), (7.3), $Y$ must be of the form as in (8.20).

\section{Summary and remarks}

Let $\mathscr{P}$ be the set of descendents of $\Gamma$ and $w(\mathscr{P})=\{w(Y): Y \in \mathscr{P}\}$. In the table below, we list $w(\mathscr{P})$ for each of the 15 families of graphs with classical parameters.

\begin{tabular}{lcc}
\hline \multicolumn{1}{c}{$\Gamma$} & $\#$ & $w(\mathscr{P}) \backslash\{0, d\}$ \\
\hline$J(\nu, d)(\nu \geqslant 2 d)$ & $(7.1)$ & $\{1,2, \ldots, d-1\}$ \\
$H(d, \ell)(\ell \geqslant 2)$ & $(7.2)$ & $\{1,2, \ldots, d-1\}$ \\
$J_{q}(\nu, d)(\nu \geqslant 2 d)$ & $(7.3)$ & $\{1,2, \ldots, d-1\}$ \\
$\operatorname{Bil}_{q}(d, e)(e \geqslant d)$ & $(7.4)$ & $\{1,2, \ldots, d-1\}$ \\
Dual polar graph & $(7.5)$ & $\{1,2, \ldots, d-1\}$ \\
\hline Doob $\left(d_{1}, d_{2}\right)\left(d=2 d_{1}+d_{2}\right)$ & $(8.2)$ & $\{1,2, \ldots, d-1\}$ \\
$\operatorname{Hem}_{d}(q)(q:$ odd $)$ & $(8.4)$ & $\{1,2, \ldots, d-1\}$ \\
$\tilde{J}_{q}(2 d+1, d)$ & $(8.20)$ & $\{1,2, \ldots, d-1\}$ \\
\hline$\frac{1}{2} H(n, 2)$ & $(8.3)$ & $\{1, d-1\}(n=2 d), \emptyset(n=2 d+1)$ \\
$\operatorname{Her}(d, \ell)$ & $(8.5)$ & $\emptyset$ \\
$\operatorname{Alt}(n, \ell)$ & $(8.6)$ & $\{1, d-1\}(n=2 d), \emptyset(n=2 d+1)$ \\
$\operatorname{Quad}(n-1, \ell)$ & $(8.7)$ & $\{1, d-1\}(n=2 d), \emptyset(n=2 d+1)$ \\
$U(2 d, \ell)$ & $(8.8)$ & $\emptyset$ \\
$D_{n, n}(\ell)$ & $(8.11)$ & $\{1, d-1\}(n=2 d), \emptyset(n=2 d+1)$ \\
$\operatorname{Ust}_{n-1}(\ell)(\ell:$ odd $)$ & $(8.15)$ & $\{1, d-1\}(n=2 d), \emptyset(n=2 d+1)$ \\
\hline
\end{tabular}

Note that the 5 families of the first group in the table are associated with regular semilattices, and that the 3 families of the second group have the classical parameters 
of graphs belonging to the first group. It should be remarked however that, for every $i$ $(0<i<d)$, the graphs in the second group possess pairs of vertices at distance $i$ which are not contained in any descendent with width $i$, with the exception of $(\Gamma, i)=\left(\operatorname{Hem}_{d}(q), 1\right)$.

In (4.5) we posed the problem of determining the filter $V_{[\Gamma]}$ of the poset $\mathscr{L}$ generated by the isomorphism class $[\Gamma]$. We end this section with describing $V_{[\Gamma]}$ for some examples where $\Gamma$ has classical parameters $(d, q, \alpha, \beta)$ with $q=1$ or $q<-1$. The distance-regular graphs with classical parameters $(d, 1, \alpha, \beta)(d \geqslant 3)$ are known: the Johnson graphs, Hamming graphs, Doob graphs, halved cubes and the Gosset graph; see e.g., [3, Theorem 6.1.1]. By virtue of (6.4), (7.1), (7.2), (8.2) and (8.3), we have the following table:

\begin{tabular}{ll}
\hline \multicolumn{1}{c}{$\Gamma$} & $V_{[\Gamma]}$ \\
\hline$J(\nu, d)(\nu \geqslant 2 d)$ & $\{[J(\nu+i, d+i)]: 0 \leqslant i \leqslant \nu-2 d\}$ \\
$H(d, \ell)(\ell \neq 4)$ & $\{[H(e, \ell)]: e \geqslant d\}$ \\
$H(d, 4)$ & $\{[H(e, 4)]: e \geqslant d\} \cup\left\{\left[\operatorname{Doob}\left(d_{1}, d_{2}\right)\right]: d_{2} \geqslant d\right\}$ \\
$\operatorname{Doob}\left(d_{1}, d_{2}\right)$ & $\left\{\left[\operatorname{Doob}\left(e_{1}, e_{2}\right)\right]: e_{1} \geqslant d_{1}, e_{2} \geqslant d_{1}\right\}$ \\
$\frac{1}{2} H(2 d, 2)$ & $\left\{\left[\frac{1}{2} H(2 d, 2)\right]\right\}$ \\
$\frac{1}{2} H(2 d+1,2)$ & $\left\{\left[\frac{1}{2} H(2 d+1,2)\right],\left[\frac{1}{2} H(2 d+2,2)\right]\right\}$ \\
\hline
\end{tabular}

Weng [43] showed that if a distance-regular graph $\Delta$ having classical parameters $(d, q, \alpha, \beta)$ satisfies $d \geqslant 4, q<-1, a_{1} \neq 0, c_{2}>1$ then either (i) $\Delta=\operatorname{Her}(d, \ell)(q=-\ell)$; (ii) $\Delta=U(2 d, \ell)(q=-\ell)$; or (iii) $\alpha=(q-1) / 2, \beta=-\left(1+q^{d}\right) / 2$ and $-q$ is a power of an odd prime. Hence, by $(6.4),(8.5),(8.8)$ it follows that $[\operatorname{Her}(d, \ell)],[U(2 d, \ell)]$ are maximal elements in $\mathscr{L}$ for all $d \geqslant 3$, so that the filter generated by any of them is a singleton. It would be interesting if the poset $\mathscr{L}$ is of some use, e.g., in the classification of distance-regular graphs $\Gamma$ with $\iota(\Gamma)=\iota\left(J_{q}(2 d+1, d)\right)=\iota\left(\tilde{J}_{q}(2 d+1, d)\right)$.

\section{A The list of parameter arrays}

We display the parameter arrays of Leonard systems. The data in (A.1) is taken from [40], but the presentation is changed so as to be consistent with the notation in $[2,34,35,36]$.

(A.1) Theorem([40, Theorem 5.16]). Let $\Phi$ be the Leonard system from (3.1) and let $p(\Phi)=\left(\left\{\theta_{i}\right\}_{i=0}^{d} ;\left\{\theta_{i}^{*}\right\}_{i=0}^{d} ;\left\{\varphi_{i}\right\}_{i=1}^{d} ;\left\{\phi_{i}\right\}_{i=1}^{d}\right)$ be as in (3.7). Then at least one of the following cases I, IA, II, IIA, IIB, IIC, III hold:

$$
\begin{gathered}
\text { (I) } p(\Phi)=p\left(\mathrm{I} ; q, h, h^{*}, r_{1}, r_{2}, s, s^{*}, \theta_{0}, \theta_{0}^{*}, d\right) \text { where } r_{1} r_{2}=s s^{*} q^{d+1}, \\
\theta_{i}=\theta_{0}+h\left(1-q^{i}\right)\left(1-s q^{i+1}\right) q^{-i} \\
\theta_{i}^{*}=\theta_{0}^{*}+h^{*}\left(1-q^{i}\right)\left(1-s^{*} q^{i+1}\right) q^{-i}
\end{gathered}
$$


for $0 \leqslant i \leqslant d$, and

$$
\begin{aligned}
\varphi_{i} & =h h^{*} q^{1-2 i}\left(1-q^{i}\right)\left(1-q^{i-d-1}\right)\left(1-r_{1} q^{i}\right)\left(1-r_{2} q^{i}\right), \\
\phi_{i} & = \begin{cases}h h^{*} q^{1-2 i}\left(1-q^{i}\right)\left(1-q^{i-d-1}\right)\left(r_{1}-s^{*} q^{i}\right)\left(r_{2}-s^{*} q^{i}\right) / s^{*} & \text { if } s^{*} \neq 0, \\
h h^{*} q^{d+2-2 i}\left(1-q^{i}\right)\left(1-q^{i-d-1}\right)\left(s-r_{1} q^{i-d-1}-r_{2} q^{i-d-1}\right) & \text { if } s^{*}=0\end{cases}
\end{aligned}
$$

for $1 \leqslant i \leqslant d$

(IA) $p(\Phi)=p\left(\mathrm{IA} ; q, h^{*}, r, s, \theta_{0}, \theta_{0}^{*}, d\right)$ where

$$
\begin{aligned}
\theta_{i} & =\theta_{0}-s q\left(1-q^{i}\right), \\
\theta_{i}^{*} & =\theta_{0}^{*}+h^{*}\left(1-q^{i}\right) q^{-i}
\end{aligned}
$$

for $0 \leqslant i \leqslant d$, and

$$
\begin{aligned}
& \varphi_{i}=-r h^{*} q^{1-i}\left(1-q^{i}\right)\left(1-q^{i-d-1}\right), \\
& \phi_{i}=h^{*} q^{d+2-2 i}\left(1-q^{i}\right)\left(1-q^{i-d-1}\right)\left(s-r q^{i-d-1}\right)
\end{aligned}
$$

for $1 \leqslant i \leqslant d$

(II) $p(\Phi)=p\left(\mathrm{II} ; h, h^{*}, r_{1}, r_{2}, s, s^{*}, \theta_{0}, \theta_{0}^{*}, d\right)$ where $r_{1}+r_{2}=s+s^{*}+d+1$,

$$
\begin{gathered}
\theta_{i}=\theta_{0}+h i(i+1+s), \\
\theta_{i}^{*}=\theta_{0}^{*}+h^{*} i\left(i+1+s^{*}\right)
\end{gathered}
$$

for $0 \leqslant i \leqslant d$, and

$$
\begin{aligned}
\varphi_{i} & =h h^{*} i(i-d-1)\left(i+r_{1}\right)\left(i+r_{2}\right), \\
\phi_{i} & =h h^{*} i(i-d-1)\left(i+s^{*}-r_{1}\right)\left(i+s^{*}-r_{2}\right)
\end{aligned}
$$

for $1 \leqslant i \leqslant d$

(IIA) $p(\Phi)=p\left(\mathrm{IIA} ; h, r, s, s^{*}, \theta_{0}, \theta_{0}^{*}, d\right)$ where

$$
\begin{aligned}
& \theta_{i}=\theta_{0}+h i(i+1+s), \\
& \theta_{i}^{*}=\theta_{0}^{*}+s^{*} i
\end{aligned}
$$

for $0 \leqslant i \leqslant d$, and

$$
\begin{aligned}
& \varphi_{i}=h s^{*} i(i-d-1)(i+r), \\
& \phi_{i}=h s^{*} i(i-d-1)(i+r-s-d-1)
\end{aligned}
$$

for $1 \leqslant i \leqslant d$ 
(IIB) $p(\Phi)=p\left(\mathrm{IIB} ; h^{*}, r, s, s^{*}, \theta_{0}, \theta_{0}^{*}, d\right)$ where

$$
\begin{aligned}
\theta_{i} & =\theta_{0}+s i \\
\theta_{i}^{*} & =\theta_{0}^{*}+h^{*} i\left(i+1+s^{*}\right)
\end{aligned}
$$

for $0 \leqslant i \leqslant d$, and

$$
\begin{aligned}
\varphi_{i} & =h^{*} \operatorname{si}(i-d-1)(i+r), \\
\phi_{i} & =-h^{*} \operatorname{si}(i-d-1)\left(i+s^{*}-r\right)
\end{aligned}
$$

for $1 \leqslant i \leqslant d$.

(IIC) $p(\Phi)=p\left(\mathrm{IIC} ; r, s, s^{*}, \theta_{0}, \theta_{0}^{*}, d\right)$ where

$$
\begin{aligned}
\theta_{i} & =\theta_{0}+s i, \\
\theta_{i}^{*} & =\theta_{0}^{*}+s^{*} i
\end{aligned}
$$

for $0 \leqslant i \leqslant d$, and

$$
\begin{aligned}
\varphi_{i} & =r i(i-d-1), \\
\phi_{i} & =\left(r-s s^{*}\right) i(i-d-1)
\end{aligned}
$$

for $1 \leqslant i \leqslant d$

(III) $p(\Phi)=p\left(\mathrm{III} ; h, h^{*}, r_{1}, r_{2}, s, s^{*}, \theta_{0}, \theta_{0}^{*}, d\right)$ where $r_{1}+r_{2}=-s-s^{*}+d+1$,

$$
\begin{aligned}
\theta_{i} & =\theta_{0}+h\left(s-1+(1-s+2 i)(-1)^{i}\right), \\
\theta_{i}^{*} & =\theta_{0}^{*}+h^{*}\left(s^{*}-1+\left(1-s^{*}+2 i\right)(-1)^{i}\right)
\end{aligned}
$$

for $0 \leqslant i \leqslant d$, and

$$
\begin{gathered}
\varphi_{i}= \begin{cases}-4 h h^{*} i\left(i+r_{1}\right) & \text { if } i \text { even, d even, } \\
-4 h h^{*}(i-d-1)\left(i+r_{2}\right) & \text { if } i \text { odd, } d \text { even, } \\
-4 h h^{*} i(i-d-1) & \text { if } i \text { even, } d \text { odd, } \\
-4 h h^{*}\left(i+r_{1}\right)\left(i+r_{2}\right) & \text { if } i \text { odd, } d \text { odd, }\end{cases} \\
\phi_{i}= \begin{cases}4 h h^{*} i\left(i-s^{*}-r_{1}\right) & \text { if } i \text { even, } d \text { even, } \\
4 h h^{*}(i-d-1)\left(i-s^{*}-r_{2}\right) & \text { if } i \text { odd, } d \text { even, } \\
-4 h h^{*} i(i-d-1) & \text { if } i \text { even, } d \text { odd, } \\
-4 h h^{*}\left(i-s^{*}-r_{1}\right)\left(i-s^{*}-r_{2}\right) & \text { if } i \text { odd, } d \text { odd }\end{cases}
\end{gathered}
$$

for $1 \leqslant i \leqslant d$ 
We call an array $p(\cdot ; \cdots)$ in (A.1) feasible if it is the parameter array of an actual Leonard system. See [40, Examples 5.3-5.15] for the explicit feasibility conditions of these arrays. The following is essentially ${ }^{12}$ given (without proof) in [34, Lemma 2.4] and can be read off the proof of [40, Theorem 5.16]:

(A.2) Proposition. Referring to (A.1), (i)-(iv) hold below:

(i) The following arrays are equal:

$$
\begin{aligned}
& p\left(\mathrm{I} ; q, h, h^{*}, r_{1}, r_{2}, s, s^{*}, \theta_{0}, \theta_{0}^{*}, d\right), \\
& p\left(\mathrm{I} ; q, h, h^{*}, r_{2}, r_{1}, s, s^{*}, \theta_{0}, \theta_{0}^{*}, d\right) .
\end{aligned}
$$

(ii) If $s s^{*} \neq 0$ then the following arrays are equal:

$$
\begin{aligned}
& p\left(\mathrm{I} ; q, h, h^{*}, r_{1}, r_{2}, s, s^{*}, \theta_{0}, \theta_{0}^{*}, d\right), \\
& p\left(\mathrm{I} ; q^{-1}, h s q, h^{*} s^{*} q, r_{1}^{-1}, r_{2}^{-1}, s^{-1}, s^{*-1}, \theta_{0}, \theta_{0}^{*}, d\right) .
\end{aligned}
$$

(iii) The following arrays are equal:

$$
\begin{aligned}
& p\left(\mathrm{II} ; h, h^{*}, r_{1}, r_{2}, s, s^{*}, \theta_{0}, \theta_{0}^{*}, d\right), \\
& p\left(\mathrm{II} ; h, h^{*}, r_{2}, r_{1}, s, s^{*}, \theta_{0}, \theta_{0}^{*}, d\right) .
\end{aligned}
$$

(iv) If $d$ is odd then the following arrays are equal:

$$
\begin{aligned}
& p\left(\mathrm{III} ; h, h^{*}, r_{1}, r_{2}, s, s^{*}, \theta_{0}, \theta_{0}^{*}, d\right), \\
& p\left(\mathrm{III} ; h, h^{*}, r_{2}, r_{1}, s, s^{*}, \theta_{0}, \theta_{0}^{*}, d\right) .
\end{aligned}
$$

Moreover, there is no equality among the feasible arrays $p(\cdot ; \cdots)$ with $d \geqslant 3$, other than (i)-(iv) above (and combinations of (i), (ii)).

Finally, we describe the parameter arrays of the $\rho$-descendents of $\Phi$ :

(A.3) Theorem([33, Theorem 6.9]). Let $\Phi$ be the Leonard system (3.1) and let $p(\Phi)$ be given as in (A.1). Let $\Phi^{\prime}$ be a Leonard system with diameter $d^{\prime} \leqslant d$. Given an integer $\rho$ $\left(0 \leqslant \rho \leqslant d-d^{\prime}\right), \Phi^{\prime}$ is a $\rho$-descendent of $\Phi$ precisely when $p\left(\Phi^{\prime}\right)$ takes the following form:

Case I:

$$
p\left(\Phi^{\prime}\right)=p\left(\mathrm{I} ; q, h^{\prime}, h^{* \prime}, r_{1} q^{\rho}, r_{2} q^{\rho}, s q^{d-d^{\prime}}, s^{*} q^{2 \rho}, \theta_{0}^{\prime}, \theta_{0}^{* \prime}, d^{\prime}\right) .
$$

Case IA:

$$
p\left(\Phi^{\prime}\right)=p\left(\mathrm{IA} ; q, h^{* \prime}, r^{\prime}, s^{\prime}, \theta_{0}^{\prime}, \theta_{0}^{* \prime}, d^{\prime}\right) \quad \text { where } \quad s^{\prime} / r^{\prime}=q^{d-d^{\prime}-\rho} s / r .
$$

\footnotetext{
${ }^{12}$ The scalars $\varphi_{i}, \phi_{i}$ first appeared in [38]. Hence we reproduce [34, Lemma 2.4] in terms of $p(\Phi)$ for accuracy purposes.
} 
Case II:

$$
p\left(\Phi^{\prime}\right)=p\left(\mathrm{II} ; h^{\prime}, h^{* \prime}, r_{1}+\rho, r_{2}+\rho, s+d-d^{\prime}, s^{*}+2 \rho, \theta_{0}^{\prime}, \theta_{0}^{* \prime}, d^{\prime}\right) .
$$

Case IIA:

$$
p\left(\Phi^{\prime}\right)=p\left(\mathrm{IIA} ; h^{\prime}, r+\rho, s+d-d^{\prime}, s^{* \prime}, \theta_{0}^{\prime}, \theta_{0}^{* \prime}, d^{\prime}\right) .
$$

Case IIB:

$$
p\left(\Phi^{\prime}\right)=p\left(\mathrm{IIB} ; h^{* \prime}, r+\rho, s^{\prime}, s^{*}+2 \rho, \theta_{0}^{\prime}, \theta_{0}^{* \prime}, d^{\prime}\right) .
$$

Case IIC:

$$
p\left(\Phi^{\prime}\right)=p\left(\mathrm{IIC} ; r^{\prime}, s^{\prime}, s^{* \prime}, \theta_{0}^{\prime}, \theta_{0}^{* \prime}, d^{\prime}\right) \quad \text { where } \quad s^{\prime} s^{* \prime} / r^{\prime}=s s^{*} / r .
$$

Case III, d even, d' even, $\rho$ even; or Case III, $d$ odd, $d^{\prime}$ odd, $\rho$ even:

$$
p\left(\Phi^{\prime}\right)=p\left(\mathrm{III} ; h^{\prime}, h^{* \prime}, r_{1}+\rho, r_{2}+\rho, s-d+d^{\prime}, s^{*}-2 \rho, \theta_{0}^{\prime}, \theta_{0}^{* \prime}, d^{\prime}\right) .
$$

Case III, d even, d' even, $\rho$ odd:

$$
p\left(\Phi^{\prime}\right)=p\left(\mathrm{III} ; h^{\prime}, h^{* \prime}, r_{2}+\rho, r_{1}+\rho, s-d+d^{\prime}, s^{*}-2 \rho, \theta_{0}^{\prime}, \theta_{0}^{* \prime}, d^{\prime}\right) .
$$

Case III, $d$ even, $d^{\prime}=1$ :

$$
p\left(\Phi^{\prime}\right)=p\left(\mathrm{IIC} ; r^{\prime}, s^{\prime}, s^{* \prime}, \theta_{0}^{\prime}, \theta_{0}^{* \prime}, 1\right) \quad \text { where } \quad s^{\prime} s^{* \prime} / r^{\prime}=1-\phi_{\rho+1} / \varphi_{\rho+1} .
$$

Case III, $d$ even, $d^{\prime}$ odd $\geqslant 3$; or Case III, $d$ odd, either $d^{\prime}$ even or $\rho$ odd: Does not occur.

Acknowledgments. The author would like to thank Tatsuro Ito, Jack Koolen, Bill Martin and Paul Terwilliger for valuable discussions and comments, and to Frédéric Vanhove for sending him a preprint. HT is indebted to the anonymous referee for his/her careful reading of the paper. HT also wishes to thank the Department of Mathematics at the University of Wisconsin-Madison for its hospitality throughout the period in which this paper was written. Support from the JSPS Excellent Young Researchers Overseas Visit Program is also gratefully acknowledged.

\section{References}

[1] S. Bang, T. Fujisaki and J. H. Koolen, The spectra of the local graphs of the twisted Grassmann graphs, European J. Combin. 30 (2009) 638-654.

[2] E. Bannai and T. Ito, Algebraic combinatorics I: Association schemes, Benjamin/Cummings, Menlo Park, CA, 1984. 
[3] A. E. Brouwer, A. M. Cohen and A. Neumaier, Distance-regular graphs, SpringerVerlag, Berlin, 1989.

[4] A. E. Brouwer, C. D. Godsil, J. H. Koolen and W. J. Martin, Width and dual width of subsets in polynomial association schemes, J. Combin. Theory Ser. A 102 (2003) $255-271$.

[5] A. E. Brouwer and J. Hemmeter, A new family of distance-regular graphs and the $\{0,1,2\}$-cliques in dual polar graphs, European J. Combin. 13 (1992) 71-79.

[6] A. E. Brouwer, J. Hemmeter and A. Woldar, The complete list of maximal cliques of $\operatorname{Quad}(n, q), q$ odd, European J. Combin. 16 (1995) 107-110.

[7] J. S. Caughman, IV, The Terwilliger algebras of bipartite $P$ - and $Q$-polynomial schemes, Discrete Math. 196 (1999) 65-95.

[8] J. S. Caughman, IV, The last subconstituent of a bipartite $Q$-polynomial distanceregular graph, European J. Combin. 24 (2003) 459-470.

[9] J. S. Caughman, IV, E. J. Hart and J. Ma, The last subconstituent of the Hemmeter graph, Discrete Math. 308 (2008) 3056-3060.

[10] D. R. Cerzo, Structure of thin irreducible modules of a $Q$-polynomial distance-regular graph, Linear Algebra Appl. 433 (2010) 1573-1613; arXiv:1003.5368.

[11] C. W. Curtis and I. Reiner, Methods of representation theory, Vol. I, John Wiley \& Sons, New York, 1990.

[12] E. R. van Dam and J. H. Koolen, A new family of distance-regular graphs with unbounded diameter, Invent. Math. 162 (2005) 189-193.

[13] P. Delsarte, Association schemes and t-designs in regular semilattices, J. Combin. Theory Ser. A 20 (1976) 230-243.

[14] T. Fujisaki, J. H. Koolen and M. Tagami, Some properties of the twisted Grassmann graphs, Innov. Incidence Geom. 3 (2006) 81-87.

[15] C. D. Godsil, Algebraic combinatorics, Chapman \& Hall, New York, 1993.

[16] J. Hemmeter, Distance-regular graphs and halved graphs, European J. Combin. 7 (1986) 119-129.

[17] J. Hemmeter, The large cliques in the graph of quadratic forms, European J. Combin. 9 (1988) 395-410.

[18] J. Hemmeter and A. Woldar, The complete list of maximal cliques of Quad $(n, q), q$ even, European J. Combin. 20 (1999) 81-85.

[19] A. Hiraki, Distance-regular graph with $c_{2}>1$ and $a_{1}=0<a_{2}$, Graphs Combin. 25 (2009) 65-79.

[20] R. Hosoya and H. Suzuki, Tight distance-regular graphs with respect to subsets, European J. Combin. 28 (2007) 61-74.

[21] R. Koekoek, P. A. Lesky and R. F. Swarttouw, Hypergeometric orthogonal polynomials and their $q$-analogues, Springer-Verlag, Berlin, 2010. 
[22] J. H. Koolen, W. S. Lee and W. J. Martin, Characterizing completely regular codes from an algebraic viewpoint, in: R. Brualdi et al. (Eds.), Combinatorics and Graphs, Contemporary Mathematics, vol. 531, American Mathematical Society, Providence, RI, 2010, pp. 223-242; arXiv:0911.1828.

[23] E. W. Lambeck, Contributions to the theory of distance regular graphs, Ph.D. Thesis, Eindhoven University of Technology, Eindhoven, The Netherlands, 1990.

[24] D. A. Leonard, Orthogonal polynomials, duality and association schemes, SIAM J. Math. Anal. 13 (1982) 656-663.

[25] W. J. Martin and H. Tanaka, Commutative association schemes, European J. Combin. 30 (2009) 1497-1525; arXiv:0811.2475.

[26] A. Munemasa, D. V. Pasechnik and S. V. Shpectorov, The automorphism group and the convex subgraphs of the quadratic forms graph in characteristic 2, J. Algebraic Combin. 2 (1993) 411-419.

[27] A. Munemasa and V. D. Tonchev, The twisted Grassmann graph is the block graph of a design, preprint; arXiv:0906.4509.

[28] V. Pepe, L. Storme and F. Vanhove, Theorems of Erdős-Ko-Rado type in polar spaces, J. Combin. Theory Ser. A 118 (2011) 1291-1312.

[29] R. P. Stanley, Enumerative combinatorics, Vol. 1, Cambridge University Press, Cambridge, 1997.

[30] H. Suzuki, The Terwilliger algebra associated with a set of vertices in a distanceregular graph, J. Algebraic Combin. 22 (2005) 5-38.

[31] H. Tanaka, Classification of subsets with minimal width and dual width in Grassmann, bilinear forms and dual polar graphs, J. Combin. Theory, Ser. A 113 (2006) 903-910.

[32] H. Tanaka, New proofs of the Assmus-Mattson theorem based on the Terwilliger algebra, European J. Combin. 30 (2009) 736-746; arXiv:math/0612740.

[33] H. Tanaka, A bilinear form relating two Leonard systems, Linear Algebra Appl. 431 (2009) 1726-1739; arXiv:0807.0385.

[34] P. Terwilliger, The subconstituent algebra of an association scheme I, J. Algebraic Combin. 1 (1992) 363-388.

[35] P. Terwilliger, The subconstituent algebra of an association scheme II, J. Algebraic Combin. 2 (1993) 73-103.

[36] P. Terwilliger, The subconstituent algebra of an association scheme III, J. Algebraic Combin. 2 (1993) 177-210.

[37] P. Terwilliger, Quantum matroids, in: E. Bannai and A. Munemasa (Eds.), Progress in algebraic combinatorics, Advanced Studies in Pure Mathematics, vol. 24, the Mathematical Society of Japan, Tokyo, 1996, pp. 323-441.

[38] P. Terwilliger, Two linear transformations each tridiagonal with respect to an eigenbasis of the other, Linear Algebra Appl. 330 (2001) 149-203; arXiv:math/0406555. 
[39] P. Terwilliger, Leonard pairs and the $q$-Racah polynomials, Linear Algebra Appl. 387 (2004) 235-276; arXiv:math/0306301.

[40] P. Terwilliger, Two linear transformations each tridiagonal with respect to an eigenbasis of the other; comments on the parameter array, Des. Codes Cryptogr. 34 (2005) 307-332; arXiv:math/0306291.

[41] P. Terwilliger, $Q$-polynomial distance-regular graphs containing a singular line with cardinality at least 3 , unpublished manuscript.

[42] C.-W. Weng, Weak-geodetically closed subgraphs in distance-regular graphs, Graphs Combin. 14 (1998) 275-304.

[43] C.-W. Weng, Classical distance-regular graphs of negative type, J. Combin. Theory Ser. B 76 (1999) 93-116. 